\title{
Estimation of surface shortwave radiation components under all sky conditions: Modeling and sensitivity analysis
}

\author{
Ling Chen a , Guangjian Yan ${ }^{\text {a,* }}$, Tianxing Wang ${ }^{\text {b }}$, Huazhong Ren a , Josep Calbó c, \\ Jing Zhao a ${ }^{\text {, Richard McKenzie }}{ }^{\mathrm{d}}$ \\ a State Key Laboratory of Remote Sensing Science, School of Geography, Beijing Normal University, Beijing 100875, China \\ b Institute of Remote Sensing Applications, Chinese Academy of Sciences, Beijing 100101, China \\ c Departament de Física, Universitat de Girona, Girona, Spain \\ d National Institute of Water and Atmospheric Research, Lauder, Central Otago 9352, New Zealand
}

\section{A R T I C L E I N F O}

\section{Article history:}

Received 30 December 2011

Received in revised form 24 March 2012

Accepted 16 April 2012

Available online 17 May 2012

\section{Keywords:}

MODTRAN-CF

Hemispherical effective cloud fraction

Global sensitivity analysis

\begin{abstract}
A B S T R A C T
Clouds are the most important modulator of the amount of solar energy absorbed by the earth-atmosphere system. Traditional one-dimensional (1D) plane-parallel atmospheric radiative transfer models which use the independent pixel approximation (IPA) can only consider two extreme conditions, i.e., either cloudfree or overcast cases. In this paper, two cloud fraction related factors (hemispherical effective cloud fraction and regional cloud fraction) are calculated and incorporated into MODTRAN 4 (one of the most popular radiative transfer packages) to simulate the surface shortwave radiation components and the top-of-atmosphere (TOA) radiance for all possible solar-cloud-viewing geometries. The accuracy of this modified solar radiative transfer model (named as MODTRAN-CF) is consistent with its prototype (MODTRAN 4) which has been widely used and validated in radiative transfer modeling. Some field measurements are used to validate the superiority of MODTRAN-CF. For further understanding and simplifying of this physical model, a global sensitivity analysis (GSA) method is employed to analyze the effect of model parameters on each surface shortwave radiation component. Five parameters including solar zenith angle, surface albedo, hemispherical effective cloud fraction, ground altitude and atmospheric visibility show non-negligible impacts on almost all surface shortwave fluxes, which indicates that these five parameters should be carefully considered in the future modeling of the surface shortwave radiation fluxes. Two cloud optical thickness related parameters (cloud extinction coefficient and cloud thickness) exhibit obvious importance only under cloudy illumination condition especially with optically thin clouds. These findings on the improved model will enhance our knowledge on how to accurately model the surface shortwave radiation fluxes under all sky conditions.
\end{abstract}

(c) 2012 Elsevier Inc. All rights reserved.

\section{Introduction}

Accurate estimation of surface shortwave radiation budget is critical for various applications including agricultural meteorology, weather prediction and climate monitoring. Surface incident shortwave radiation is a significant variable of many numerical models to estimate soil moisture, evapotranspiration and photosynthesis. It is also a key factor to determine the location of solar heating systems in solar energy application. The surface solar radiation flux is mainly affected by atmospheric properties and to a lesser extent by surface conditions. Among all the atmospheric constituents, clouds are considered to be the strongest modulator of the solar radiant energy absorbed by the earth-atmosphere system due to their particular spatial and temporal variability in conjunction with their strong

\footnotetext{
* Corresponding author. Tel.: + 861058802085.

E-mail address: gjyan@bnu.edu.cn (G. Yan).
}

interaction with solar radiation (Arking, 1991; Hartmann et al., 1986; Li et al., 1995). Therefore, it is of great importance to estimate solar radiation budget under cloudy atmospheres, which are most prevalent.

So far, various noteworthy atmospheric radiative transfer models and algorithms have been proposed to estimate surface solar radiation components from satellite data. They can be classified into two broad categories: three-dimensional (3D) radiative transfer models (e.g., Cornet et al., 2010; Evans, 1998; Gu \& Liou, 2001; O'Hirok \& Gautier, 1998) and one-dimensional (1D) plane-parallel radiative transfer algorithms (e.g., Ceballos et al., 2004; Deneke et al., 2005; Diak \& Gautier, 1983; Freidenreich \& Ramaswamy, 1999; Frouin \& Chertock, 1992; Gautier \& Landsfeld, 1997; Gautier et al., 1980; Li et al., 1993; Masuda et al., 1995; Pinker \& Ewing, 1985; Pinker \& Laszlo, 1992). Using full 3D radiative transfer models may be the most accurate method to retrieve surface shortwave radiation budget under cloudy atmospheres, however, low computational efficiency makes them less suitable for operational purposes (Cahalan et al., 
2005; Marshak \& Davis, 2005). Most of the 1D algorithms use the independent pixel approximation (IPA) which treats each pixel in a satellite image independently and neglects radiative transfer effects between different atmospheric columns (pixels). Therefore, the independent pixel approximation is only suitable to estimate domainaveraged solar flux over large areas, in which the effects of horizontal transport of photons are not important (Barker, 1996; Barker et al., 1999; Cahalan et al., 1994; Wyser et al., 2002; Wyser et al., 2005; Zuidema \& Evans, 1998). For example, 3-hour global radiative flux data sets have been derived from the International Satellite Cloud Climatology Project (ISCCP) with a spatial resolution of $2.5^{\circ}$ (Zhang et al., 2004) and from the Global Energy and Water Cycle Experiment (GEWEX) with a spatial resolution of $1^{\circ}$ (Pinker \& Laszlo, 1992). Surface solar fluxes with such coarse resolutions and the existence of unresolved sub-pixel clouds may not satisfy the requirements of many land applications (Liang et al., 2010).

Recently, many researchers have estimated surface solar fluxes through the combination of $1 \mathrm{D}$ radiative transfer models and high spatial resolution data and products offered by modern sensors such as Moderate Resolution Imaging Spectroradiometer (MODIS) (e.g., Kim, 2008; Liang et al., 2006; Liu et al., 2008; Tang et al., 2006; Zheng, 2007). For example, Liang et al. (2006) developed a look-up table approach based on MODTRAN simulations to estimate the incident photosynthetically active radiation (PAR) from MODIS data. Tang et al. (2006) modified the parameterization coefficients of Masuda et al. (1995) based on the comprehensive MODTRAN simulations and then applied them in the estimation of net surface shortwave radiation (NSSR) based on MODIS data. With increasing spatial resolution, however, the 3D radiative effects caused by clouds from surrounding atmospheric columns in the partially cloudy atmosphere become more important. Based on a 3D Monte Carlo radiation model, Wyser et al. (2002) suggested that for IPA algorithms used at high spatial resolution, corrections should be applied to account for the effects of nonlocal cloud shadows and photon diffusion from neighboring columns. The main objective of the present study is to include these corrections into a classic 1D physical radiative transfer algorithm in order to improve its ability of accurately calculating the surface shortwave radiation budget from high spatial resolution remote sensing data and products.

For this purpose, we start from incorporating various solar-cloudviewing geometries, hemispherical effective cloud fraction (HECF) and regional cloud fraction (RCF) into the original solar radiative transfer equations to obtain a modified physical radiative transfer model. As we all know, better understanding of model behavior through sensitivity analysis is an indispensable step for further simplification or parameterization. To this end, one of the most accurate global sensitivity analysis (GSA) methods based on Monte Carlo sampling is addressed in this paper: the quantitative Sobol' (1993) method which is able to explicitly handle possible interactions among parameters. To our knowledge, this is the first time that GSA methods have been carried out on an atmospheric radiative transfer model.

The paper is organized as follows. Section 2 describes the modified physical model and its parameters. The methodology and results of the selected GSA method are presented in Section 3. Several case simulations and the validation results are shown in Section 4. A brief summary is given at the end of this paper.

\section{Improved radiative transfer model}

\subsection{Model description}

MODTRAN is one of the most widely used radiative transfer codes that are available for the public research community (e.g., Liang et al., 2006; Liu et al., 2008; Tang et al., 2006). It is a classic 1D model which takes into account multiple scattering in a plane-parallel vertically inhomogeneous atmosphere. Assuming the surface is Lambertian, the downward spectral flux $F\left(\mu_{i}\right)$ and TOA upwelling radiance $L\left(\mu_{i}, \mu_{v}\right.$, $\phi)$ can be calculated from the following formulas (Liang, 2004; Liang et al., 2006):

$F\left(\mu_{i}\right)=F_{0}\left(\mu_{i}\right)+\frac{r_{s} \bar{\rho}}{1-r_{s} \bar{\rho}} \mu_{i} E_{0} \gamma\left(\mu_{i}\right)$

$L\left(\mu_{i}, \mu_{v}, \phi\right)=L_{p}\left(\mu_{i}, \mu_{v}, \phi\right)+\frac{r_{s}}{1-r_{s} \bar{\rho}} \mu_{i} \frac{E_{0}}{\pi} \gamma\left(\mu_{i}\right) \gamma\left(\mu_{v}\right)$

where $\mu_{i}$ is the cosine of solar zenith angle $\theta_{i}, \mu_{v}$ is the cosine of viewing zenith angle $\theta_{v}, \phi$ is the relative azimuth angle, $F_{0}$ and $L_{p}$ are the downward flux and path radiance without any surface contribution (i.e., surface reflectance $r_{s}$ is zero), $\bar{\rho}$ is the spherical albedo of the atmosphere, $E_{0}$ is the TOA solar irradiance, and $\gamma\left(\mu_{i}\right)$ and $\gamma\left(\mu_{v}\right)$ are the total atmospheric transmittance in the solar illumination and viewing direction respectively. All radiometric variables in the above two equations are functions of wavelength.

However, the assumption of plane-parallel vertically inhomogeneous atmosphere cannot take into account partly cloudy conditions, which means that it can only consider two extreme situations: pure clear skies (i.e., cloud-free, Fig. 1A) and uniformly overcast skies (i.e., totally cloud-covered, Fig. 1B).

In fact, the real situation is much more complicated than cases mentioned above, which can be classified into four categories: clear illumination and cloudy viewing path (Fig. 2A), cloudy illumination and clear viewing path (Fig. 2B), both illumination and viewing directions are clear (Fig. 2C) and both are cloudy (Fig. 2D).

It is a rather complex problem to model solar radiative transfer in a cloudy atmosphere, especially under partially cloudy situations as
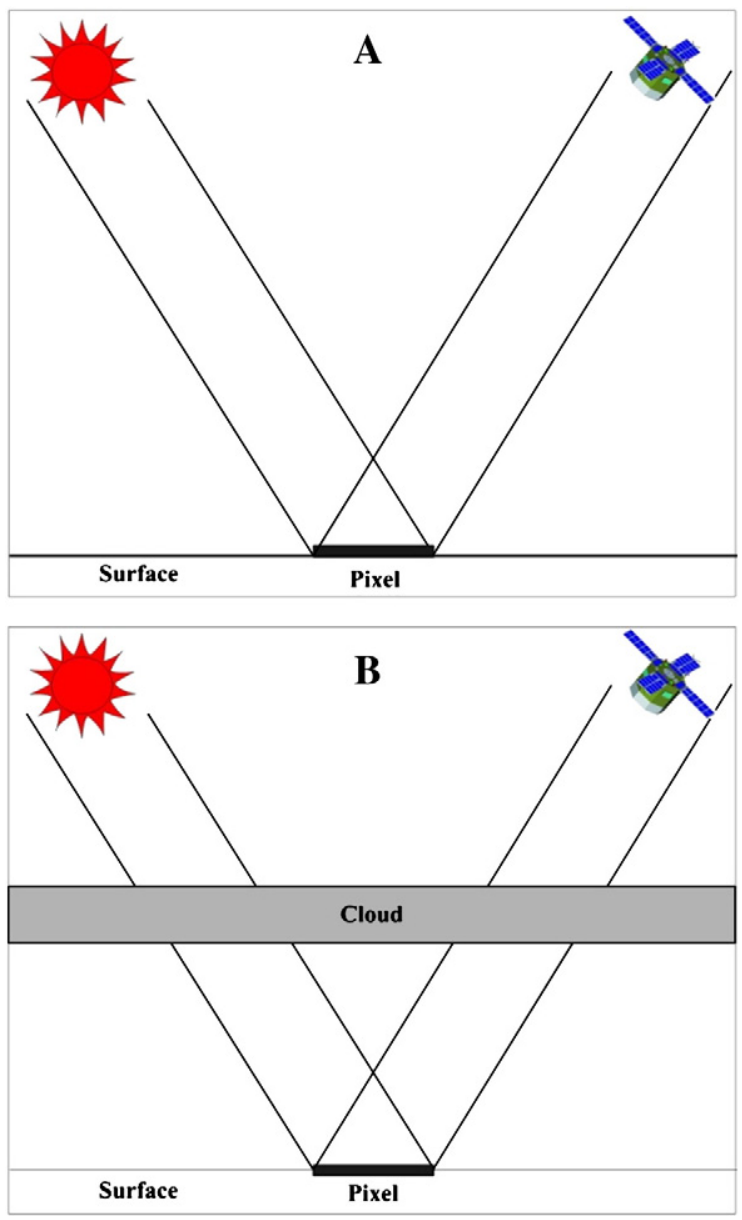

Fig. 1. Illustrations of clear and cloudy modes in MODTRAN. 


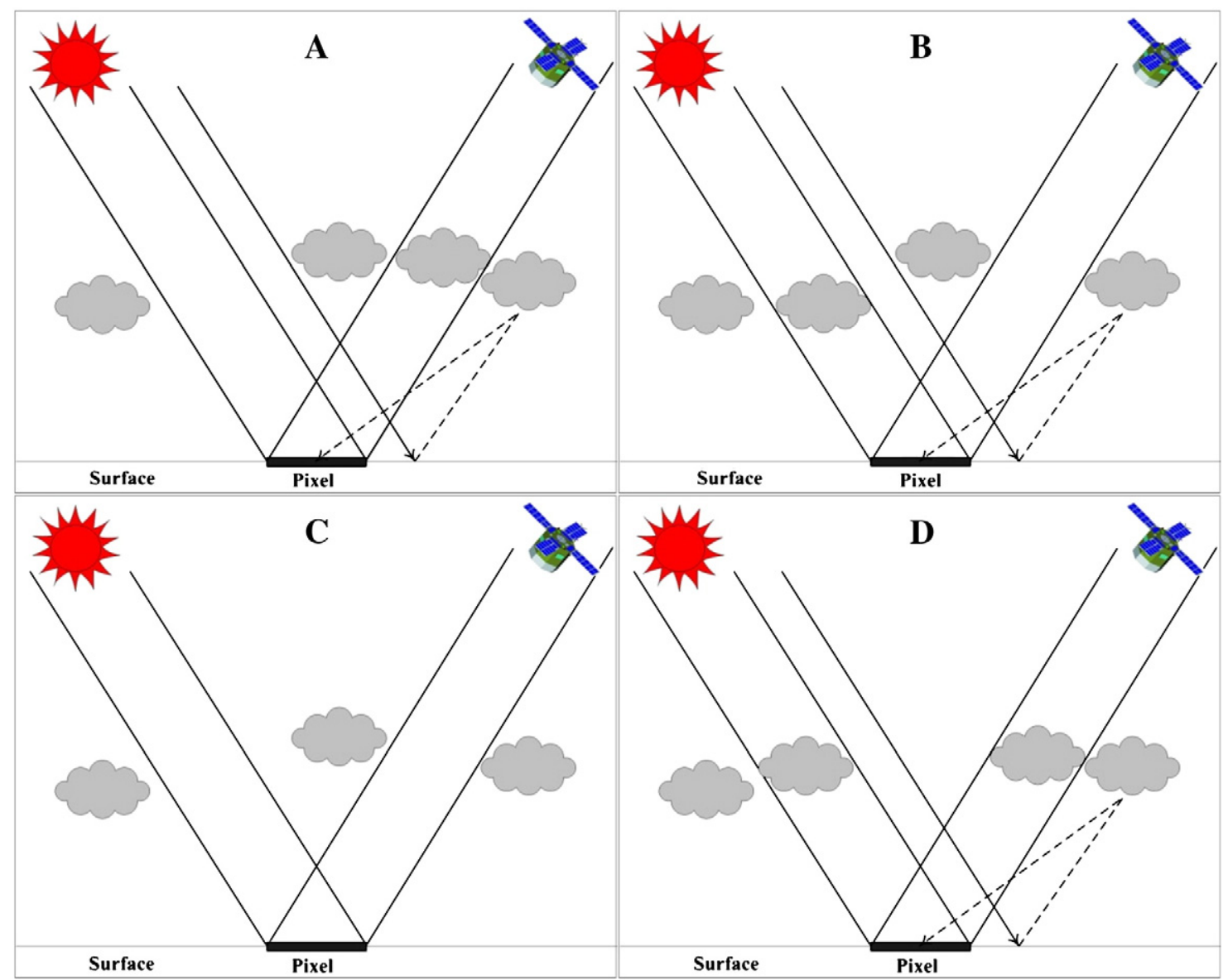

Fig. 2. Illustrations of four more realistic illumination and viewing modes.

illustrated in Fig. 2. Our main goal here is to improve the frequently used 1D plane-parallel cloud-radiation model by employing two cloud fraction related factors and all possible illumination-cloudviewing geometries under all sky conditions. Since our main calculation processes are based on MODTRAN 4 and only two cloud factors are additionally introduced, hereinafter, this modified solar radiative transfer model is referred to as MODTRAN-CF. We must note that the cloud properties, which include cloud base height, cloud extinction coefficient and cloud thickness, are identical between the surrounding areas and the ones within the illumination or viewing path at the present study.

In the following parts, the strategies to consider the partially cloudy situations for both Eqs. (1) and (2) are illustrated. Let us begin with the modifications to Eq. (1), in which some variables will then be used to formulate the TOA upwelling radiances.

\subsubsection{Surface downward flux modeling}

Two situations should be considered in the derivation of the downward flux $F_{0}$ : the illumination is under clear condition (Fig. $2 \mathrm{~A}$ and C) and the illumination is truncated by clouds (Fig. 2B and D). $F_{0}$ is composed of two parts, i.e., direct solar flux and downward diffuse flux. For the clear illumination cases, it can be expressed by:

$F_{0}=\operatorname{Dir}_{\text {clear }}+\operatorname{Dif}_{\text {cloud }} \cdot f+\operatorname{Dif}_{\text {clear }} \cdot(1-f)$

where $D i r_{\text {clear }}$ is the direct solar spectral flux retrieved by running MODTRAN in clear modes, Dif $f_{\text {clear }}$ and Dif cloud $_{\text {are }}$ are downward diffuse spectral fluxes retrieved by running MODTRAN when the surface reflectance $r_{s}$ is set to zero in clear and cloudy modes respectively, and the weighting factor $f$ is the hemispheric effective cloud fraction (HECF) for a single point or pixel.

Similarly, $F_{0}$ for cloudy illumination cases can be expressed as:

$F_{0}=\operatorname{Dir}_{\text {cloud }}+\operatorname{Dif}_{\text {cloud }} \cdot f+\operatorname{Dif}_{\text {clear }} \cdot(1-f)$

where Dir $_{\text {cloud }}$ is the direct solar spectral flux by running MODTRAN in cloudy modes.

For a Lambertian surface with reflectance of $r_{s}$, the multiple scattering part $F_{m}$ of the downward spectral flux can be derived as follows:

$F_{m}=\left[F_{0 \text { clear }} \cdot\left(1-f^{\prime}\right)+F_{0 \text { cloud }} \cdot f^{\prime}\right] \cdot \frac{r_{s} \cdot\left[(1-f) \cdot \rho_{a}+f \cdot \rho_{c}\right]}{1-r_{s} \cdot\left[\left(1-f^{\prime}\right) \cdot \rho_{a}+f^{\prime} \cdot \rho_{c}\right]}$

where $F_{0 \text { clear }}$ and $F_{0 c l o u d}$ are the downward flux without any surface contribution retrieved by running MODTRAN in clear and cloudy modes respectively, $\rho_{a}$ and $\rho_{c}$ are the spherical albedo of the clear and overcast atmosphere respectively, and $f^{\prime}$ is the regional cloud fraction (RCF) which is also referred to as nadir-view cloud fraction.

Then we get the total downward spectral flux $F$ :

$F=F_{0}+F_{m}$

Note that all the flux variables mentioned above are functions of wavelength and solar zenith angle. Surface shortwave flux components are the integrations of their corresponding spectral fluxes over the shortwave spectral range $(0.3-3.0 \mu \mathrm{m})$. Given the downward spectral flux, it is straightforward to retrieve PAR just by setting the spectral integration range to $0.4-0.7 \mu \mathrm{m}$. 


\subsubsection{TOA upwelling radiance modeling}

Similar to the surface downward flux modeling in Section 2.1.1, the path radiance $L_{p}$ should also be considered for two cases: clear viewing path (Fig. $2 \mathrm{~B}$ and $\mathrm{C}$ ) and cloudy viewing path (Fig. $2 \mathrm{~A}$ and D). In terms of the clear viewing path, $L_{p}$ is the path radiance without the surface contributions by running the MODTRAN in clear modes. Similarly, for the case of cloudy viewing path, $L_{p}$ is the path radiance without the surface contributions by running the MODTRAN in cloudy modes.

The radiative contribution of a Lambertian surface to the TOA upwelling radiance can be expressed by:

$L_{s}=\frac{r_{s} \cdot \gamma\left(\mu_{v}\right) \cdot F}{\pi}$

where $\gamma\left(\mu_{v}\right)$ is the total atmospheric transmittance (direct plus diffuse) from the surface to the sensor.

Finally, the TOA upwelling radiance $L$ can be derived as:

$L=L_{p}+L_{s}$

Note that all the radiances in the above two equations are functions of wavelength and the solar-viewing geometry. The spectral TOA radiances are weighted by the sensor response function and integrated over the spectral range of the response of each band.

The operation of MODTRAN-CF is mainly dependent on running the MODTRAN 4 package. For a given atmospheric condition and solar-cloud-viewing geometry, the four unknowns $\left(\rho_{a}, \rho_{c}, \gamma\left(\mu_{v}\right)\right.$ of clear and of cloudy viewing path) in the above equations can be easily determined by running MODTRAN 4 in its corresponding modes (clear or cloudy) for a specific surface type. HECF and RCF are the extra variables that should be set by the users. Outputs of MODTRAN-CF include all the surface shortwave flux components and TOA channel radiances of certain sensor configuration.

\subsection{Model parameters}

It is necessary to identify the most influential model parameters among all the inputs via sensitivity analysis. As mentioned above, all the parameters except HECF and RCF are included in the original MODTRAN code. HECF, which is applied to any given point or pixel on the land surface, is the fraction of hemispherical sky covered by clouds. RCF is the nadir-view cloud fraction for the hemispherical sky projected to a horizontal surface. Theoretically, the cone zenith angle of a hemisphere is $90^{\circ}$ which corresponds to an infinite horizontal surface. Practically, a finite region with radius $R$ needs to be determined before these two factors are calculated. $R$ is connected to the cone zenith angle $\alpha$ of the finite region and the cloud base height $H$ as $R=H \tan (\alpha)$. In this study, we only consider water clouds with their base height below $3 \mathrm{~km}$. Fig. 3 shows that a radius of $20 \mathrm{~km}$ is appropriate to be used as a threshold, which contributes to about $90 \%$ of the infinite hemisphere. From the pixel point of view, we often use a moving window instead, therefore the size of the moving window can be set to $40 * 40 \mathrm{~km}$.

HECF of the center pixel and RCF of the moving window can be calculated as follows (Fig. 4):

$$
\begin{aligned}
H E C F & =\sum_{i=0}^{n} \frac{s \cos ^{3} \theta_{i}}{2 \pi(1-\cos \alpha) h_{i}{ }^{2}} \\
R C F & =\frac{n s}{A}
\end{aligned}
$$

where $s$ and $A$ are the area of the pixel and the moving window respectively, $n$ is the number of nadir-view cloud pixel in this moving window, $\theta_{i}$ is the inclination angle of the nadir-view cloud pixel relative to the center pixel, $2 \pi(1-\cos \alpha)$ is the solid angle of the finite

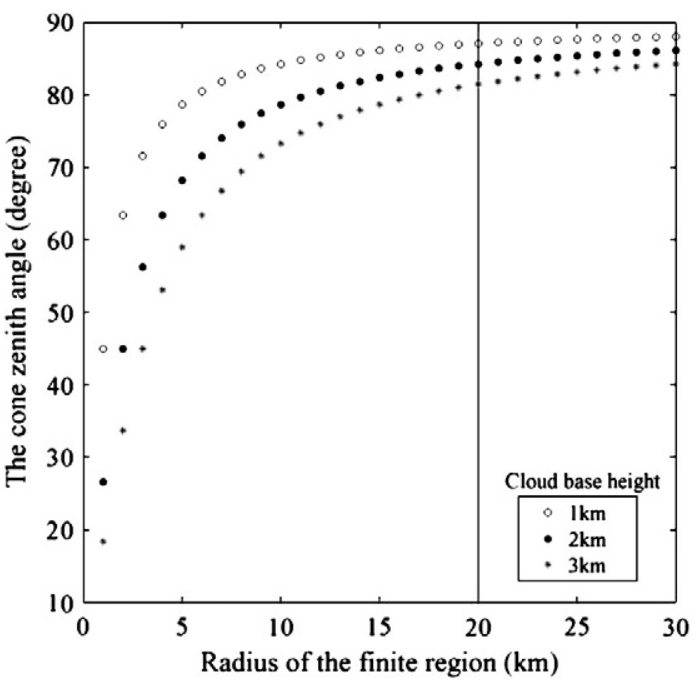

Fig. 3. Relationship between the radius of the finite region and the cone zenith angle.

hemisphere and HECF is the fraction of this solid angle filled by clouds, and $h_{i}$ is the cloud base height relative to the center pixel.

Besides the two extra parameters described above, ten other factors are chosen to participate the sensitivity analysis (Table 1). Note that only three cloud related parameters of MODTRAN are selected, others are set in default since they should be given by the cloud profile which probably is not readily available. Moreover, we consider two kinds of clouds which correspond to optically thick and thin clouds respectively. Optically thick clouds could scatter and absorb solar radiation without allowing any sunlight to penetrate through directly to the surface, i.e., surface cannot receive any direct solar flux when the illumination direction is blocked by optically thick clouds. On the other hand, direct solar radiation could be partially transmitted through optically thin clouds. We only focus on the factors which are related to the surface radiation components in our

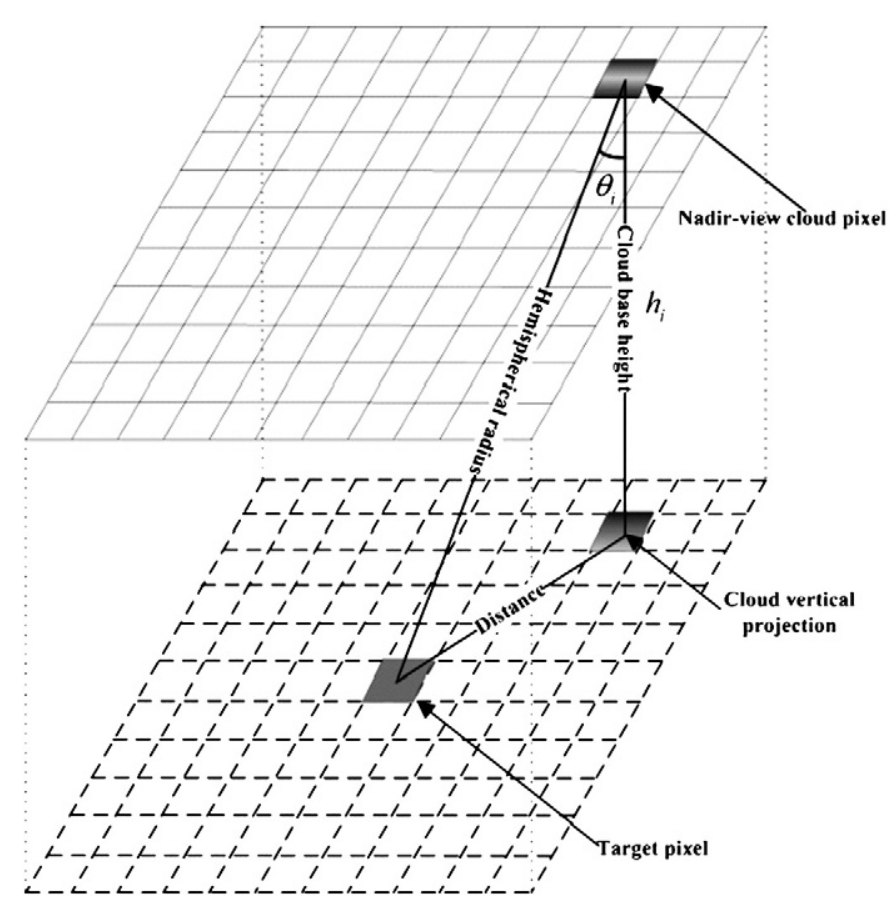

Fig. 4. Contribution of each nadir-view cloud pixel in the moving window to the HECF of the center pixel. 
Table 1

Parameters of the MODTRAN-CF model and their settings used for sensitivity analysis.

\begin{tabular}{llll}
\hline Parameter & Description & Unit & Range \\
\hline SZA & Solar zenith angle & $\circ$ & $2-74$ \\
Water & Total column water vapor & $\mathrm{g} / \mathrm{cm}^{2}$ & $0.001-1.372$ \\
Ozone & Total column ozone & $\mathrm{DU}$ & $188-564$ \\
Albedo & Land surface albedo & - & $0.02-0.9$ \\
VIS & Surface meteorological range & $\mathrm{km}$ & $2-60$ \\
$\mathrm{CO}_{2}$ & $\mathrm{CO}_{2}$ mixing ratio & $\mathrm{ppmv}$ & $360-390$ \\
$\mathrm{CTHIK}_{\mathrm{N}}$ & Cloud thickness & $\mathrm{km}$ & $(1-3) /(0.5-2)^{\mathrm{a}}$ \\
CALT & Cloud base altitude & $\mathrm{km}$ & $0.5-2.0$ \\
$\mathrm{CEXT}$ & Cloud extinction coefficient at $0.55 \mu \mathrm{m}$ & $\mathrm{km}{ }^{-1}$ & $(10-50) /(0.1-5)^{\mathrm{a}}$ \\
Alt & Ground altitude above sea level & $\mathrm{km}^{\mathrm{a}}$ & $0-6$ \\
$\mathrm{HECF}$ & Hemispherical effective cloud fraction & - & $0-1$ \\
RCF & Regional cloud fraction & - & $0-1$ \\
\hline
\end{tabular}

a Two groups of cloud thickness and extinction coefficient values are chosen for the sensitivity analysis, which correspond to optically thick clouds (opaque) and optically thin clouds (transparent) respectively.

following sensitivity analysis, so factors related to the viewing geometry are not included in Table 1 . In this paper, the default mid-latitude winter atmosphere is selected for the sensitivity analysis, the range of total column water vapor is determined with reference to its extreme values, and the range of total column ozone is set according to its default value which is $8.07 \mathrm{~g} / \mathrm{m}^{2}(\approx 376 \mathrm{DU})$. The maximum value of ground altitude has also been constrained if the default atmospheric profiles are adopted, values above $6 \mathrm{~km}$ are considered invalid.

\section{Sensitivity analysis}

\subsection{Sensitivity analysis method}

The current well-established sensitivity analysis methods can be grouped into two categories: local and global techniques (Cariboni et al., 2007). Local approaches estimate the effect of a single factor on the outputs while keeping all the others fixed at their nominal values. On the contrary, global sensitivity analysis (GSA) approaches estimate the effect of a certain factor while allowing all the others vary. The sensitivity index of a specific parameter calculated by the local sensitivity analysis is dependent on the specific values of other parameters. Thus, more and more studies tend to use GSA techniques instead of local sensitivity analysis (e.g., Confalonieri, 2011; Confalonieri et al., 2010; Drouet et al., 2011; Richter et al., 2010).

Among all the GSA methods, we select the quantitative Sobol' (1993) method to determine the key impact factors of each surface shortwave radiation components. The Sobol' method allows the simultaneous exploration of the parameters space via Monte Carlo sampling. It is a variance-based technique, which means that the variance of the model output can be decomposed into partial variances that represent the contribution of each single input (and pairs, triplets, etc.) to the overall uncertainty of the model output. The total variance $V$ of the output $y$ can be decomposed as (Confalonieri et al., 2010; Saltelli et al., 2000; Sobol', 2001):

$$
\begin{aligned}
V(y) & =\sum_{i=1}^{n} D_{i}+\sum_{i=1}^{n} \sum_{i<j}^{n} D_{i j}+\cdots+D_{12 \cdots n} \\
D_{i} & =V\left[E\left(y \mid x_{i}\right)\right] \\
D_{i j} & =V\left[E\left(y \mid x_{i}, x_{j}\right)\right]-D_{i}-D_{j}
\end{aligned}
$$

where $n$ is the number of input factors, $D_{i}$ represents the first order effect (i.e., main effect) of factor $x_{i}, D_{i j}$ represents the interaction between two factors and $D_{12} \cdots_{n}$ denotes the interactions among all factors.

Variance-based methods provide two sensitivity measures for each factor: the first order sensitivity index $S_{i}$ and the total sensitivity index $S_{t i}$ :

$$
\begin{aligned}
& S_{i}=D_{i} / V(y) \\
& S_{t i}=S_{i}+\sum_{j>i}^{n} S_{i j}+S_{1 \cdots n} \\
& S_{i j}=D_{i j} / V(y)
\end{aligned}
$$

where $S_{i}$ denotes the main contribution of parameter $x_{i}$ to the output variance only, it does not take into account the interactions among factors. $S_{t i}$ denotes the total contribution of parameter $x_{i}$ to the variance of the output, which includes the interactions between parameter $x_{i}$ and other factors. The total effects are useful to identify input factors with minor influence. If the total effect for a given input factor is negligible, then it can be fixed to any value within its range of uncertainty, and the dimensionality of input space can be reduced accordingly.

From the above two formulas, we can see that the sum of all the total sensitivity indices will not equal to one because of the interactions among factors. For the sake of consistency, the total sensitivity index of each parameter is normalized by the sum of all the total sensitivity indices, and parameters with $S^{\prime}{ }_{t i}$ lower than $1 \%$ are considered as insignificant factors in this paper:

$S_{t i}^{\prime}=\frac{S_{t i}}{\sum_{i=1}^{n} S_{t i}}$.

\subsection{Sensitivity analysis results}

In the experiment, 6656 combinations of 12 model parameters were randomly generated with the Sobol' method, using a uniform distribution between the lower and upper bounds for each parameter (Table 1), since there was no a priori information to guide the selection of the statistical distribution. Once the samples of the parameter combinations were generated by the professional sensitivity analysis software (SimLab, 2011), MODTRAN-CF with eight-stream DISORT (Stamnes \& Tsay, 1988) was run iteratively through the parameter space. The outputs of the simulations include the surface shortwave flux components and the TOA narrowband radiances for a specific sensor such as MODIS. Since our ultimate goal here is to retrieve surface shortwave fluxes through related remote sensing products, we analyzed only the sensitivity of the surface shortwave flux components to the 12 input parameters listed in Table 1.

As a primary source of surface energy balance, the downward surface shortwave radiation (DSSR) is the sum of direct and diffuse solar radiation. The partitioning of DSSR into its direct and diffuse components is needed to model the effect of topography on the spatial distribution of radiation (Dubayah, 1992), interaction of radiation with vegetation (Monteith, 1975), or penetration of radiation into the ocean (Woods et al., 1984). It is also very important in assessing the effect of solar radiation on building designs (Lam \& Li, 1996). Furthermore, the proportion of diffuse to direct solar radiation can influence factors such as forest photosynthesis and carbon cycling (Butt et al., 2010). NSSR, which is a critical factor in global and regional climatic models, is defined as the difference between the DSSR and the upward surface shortwave radiation (USSR), the latter being the portion of shortwave radiation reflected by the surface. Therefore, surface shortwave radiation can be clearly represented by five quantities: direct solar flux, downward diffuse flux, DSSR, NSSR, and USSR. The GSA results for these five quantities will be described as follows. 
A

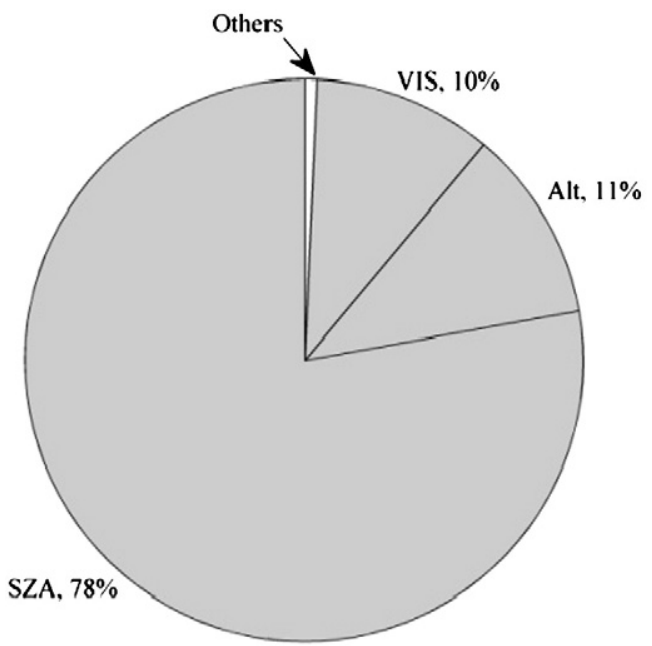

B

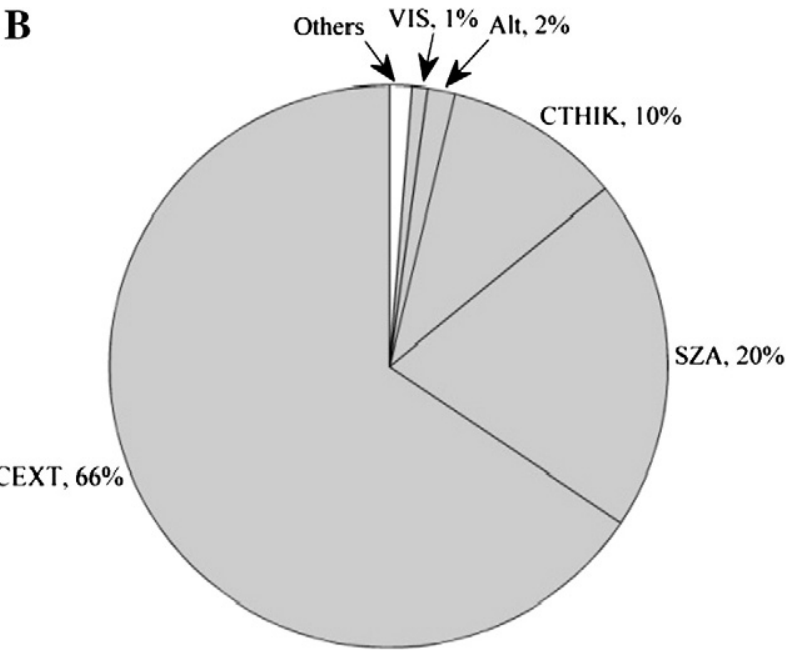

Fig. 5. Normalized total sensitivity index for direct solar flux with clear illumination path (A) and cloudy illumination path obscured by optically thin clouds (B), values lower than $1 \%$ are not listed individually (the same below).

Fig. 5 shows the GSA results of the direct solar flux which is determined only by the condition of illumination direction. For the clear illumination case, only 3 out of 12 parameters (SZA, Alt and VIS) contribute significantly to the total variance of the direct solar flux (Fig. 5A), which means that all the other parameters could be set as fixed values while using MODTRAN-CF to retrieve direct solar flux in the corresponding situations. As described above, no direct solar flux reaches the ground when the illumination direction is blocked by optically thick clouds. If the illumination path is obscured by optically thin clouds, 2 out of 3 most influential parameters (CEXT, SZA and CTHIK) are related to clouds which contribute $76 \%$ of the total variance (Fig. 5B), suggesting that the cloud effective optical depth (i.e., $\mathrm{CEXT} * \mathrm{CTHIK} / \cos (\mathrm{SZA})$ ) should be carefully quantified when the illumination direction is obscured by optically thin clouds. Fig. 6 illustrates the GSA results of surface downward diffuse radiation which is independent of whether the sun is obscured by clouds. If there are optically thick clouds in the overlying hemisphere (Fig. 6A), Albedo, SZA and HECF are the three most sensitive parameters which together contribute $89 \%$ of the variance of the downward diffuse flux. For the case of optically thin clouds (Fig. 6B), HECF becomes the most sensitive parameter, followed by SZA, CEXT and Albedo. The other three parameters with contributions greater than $1 \%$ are VIS, Alt and CTHIK.

The GSA results of the DSSR are shown in Figs. 7 and 8. SZA makes a significantly higher contribution than other parameters to the DSSR when the illumination path is clear no matter what kind of clouds
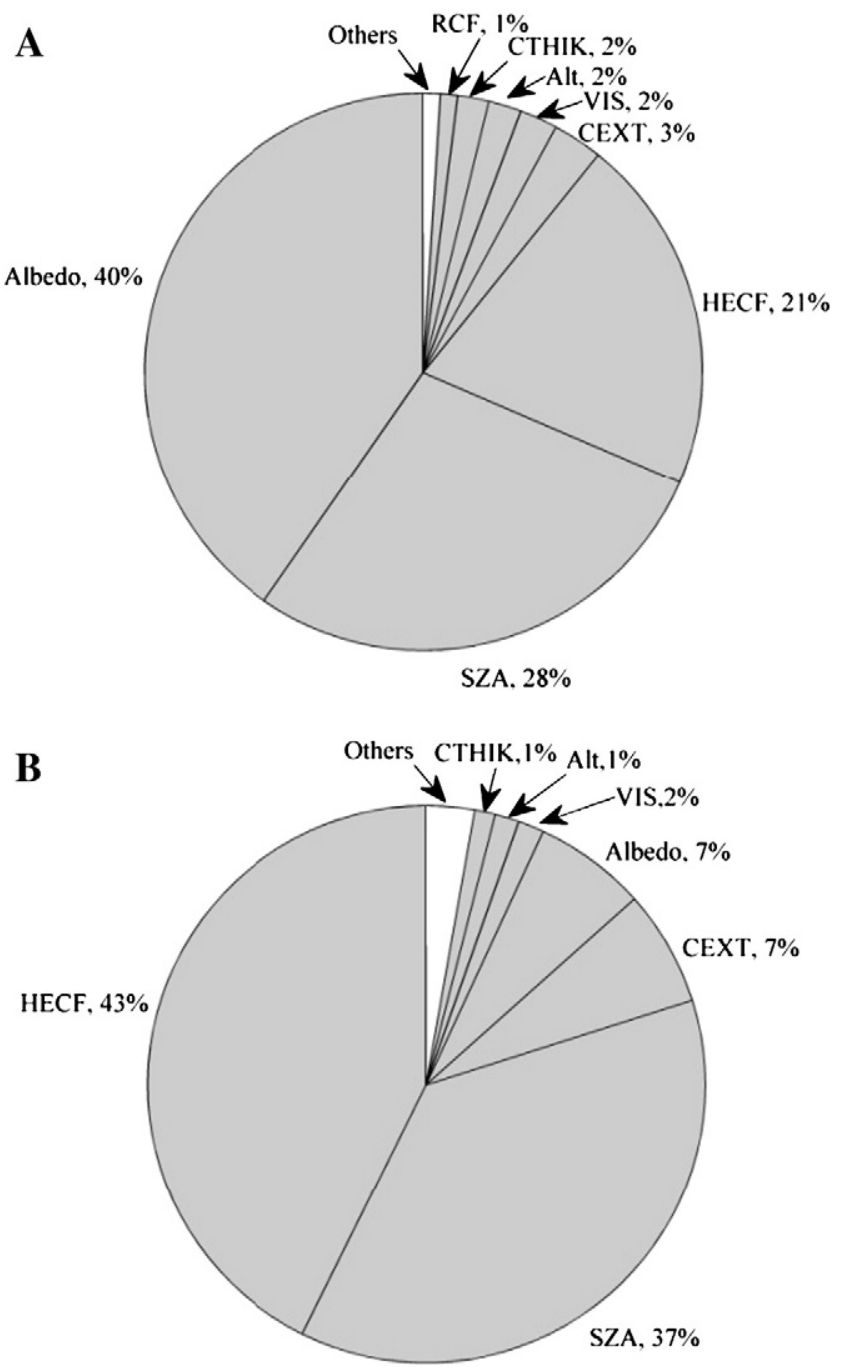

Fig. 6. Normalized total sensitivity index for downward diffuse flux with optically thick clouds (A) or optically thin clouds (B) might exist in the hemisphere.

might exist over the hemisphere (Fig. 7). This is reasonable because DSSR is mainly composed by direct solar flux which is also highly sensitive to SZA in clear illumination conditions (Fig. 5A). Among all the factors, the Sobol' method selects only five (SZA, Albedo, Alt, VIS and HECF) for the case of optically thick clouds (Fig. 7A), and six (SZA, HECF, Alt, VIS, CEXT and Albedo) for the case of optically thin clouds in which cloud related parameters (HECF and CEXT) become more important (Fig. 7B). The DSSR is composed only by the downward diffuse flux if the illumination path is obscured by optically thick clouds, so Fig. 8A is identical with Fig. 6A. Seven parameters (SZA, HECF, CEXT, Albedo, CTHIK, Alt and VIS) are determined for the case of optically thin clouds (Fig. 8B), in which the total contribution of the top three parameters (SZA, HECF, and CEXT) reaches up to $87 \%$. Albedo ranks first with the normalized total sensitivity index of $40 \%$ for optically thick clouds (Fig. 8A), but decreases to the fourth place for optically thin clouds (Fig. 8B). This reduction can be attributed to the two land surface independent fluxes including direct solar flux and the atmospheric downward diffuse flux. When the illumination direction is obscured by optically thick clouds, DSSR is composed only by the atmospheric downward diffuse flux and the surfaceatmosphere multiple scattering which is highly related to the surface albedo. When the illumination direction is obscured by optically thin clouds, DSSR can be composed by three parts including some direct solar flux, atmospheric downward diffuse flux and the multiple scattering. It can be seen that the importance of surface albedo is much 

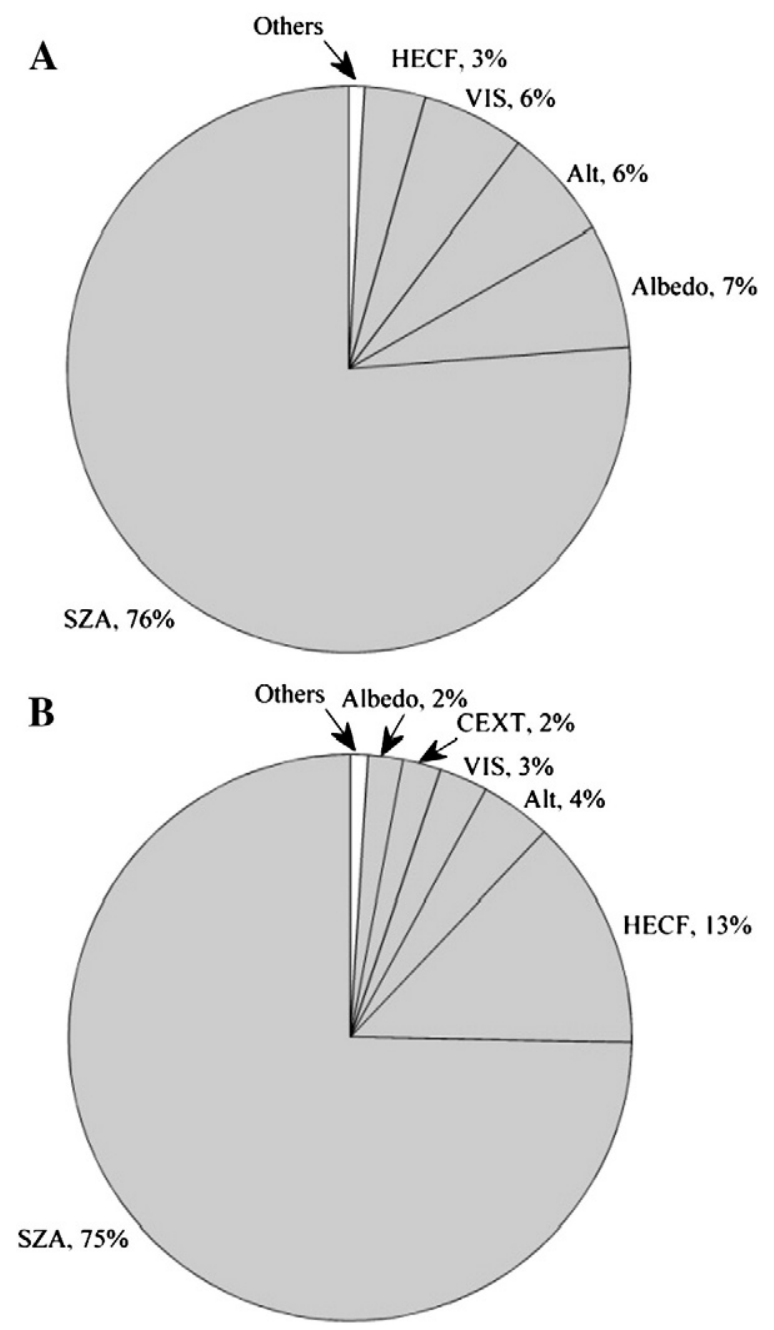

Fig. 7. Normalized total sensitivity index for DSSR with clear illumination path, and optically thick clouds (A) or optically thin clouds (B) might exist in non-illumination hemisphere.

lower because of land surface independent fluxes take much larger proportions of DSSR for this case.

As far as NSSR is concerned, SZA and Albedo are selected as the two most important parameters when the illumination path is clear (Fig. 9). The same five parameters (Albedo, SZA, HECF, Alt, and VIS) have the normalized index higher than $1 \%$ for both the optically thick and thin clouds cases. The difference is that HECF ranks lower than Alt and VIS for the case of optically thick clouds (Fig. 9A), but higher than them for the case of optically thin clouds (Fig. 9B). When the solar illumination direction is obscured by clouds, eight parameters for optically thick clouds (Fig. 10A) and five parameters for optically thin clouds (Fig. 10B) are considered as non-negligible parameters. The four most important parameters for both cases are Albedo, SZA, HECF and CEXT, which is the same as DSSR. However, because of the negative correlation between NSSR and Albedo, the importance of Albedo for optically thick clouds is lower than that for optically thin clouds, which is exactly the opposite of DSSR. The total contribution of the four parameters reaches up to $87 \%$ for optically thick clouds and $99 \%$ for optically thin clouds respectively. It can be been seen from Figs. 11 and 12 that the most important factors for USSR is the Albedo, followed by SZA. Cloud-related parameters (HECF, CEXT, or CTHIK) are more important for the cases of optically thin clouds. For the clear illumination cases, five parameters (Albedo, SZA, Alt, VIS and HECF) have the normalized index higher than $1 \%$ for optically thick cloud (Fig. 11A), and six parameters (Albedo, SZA,
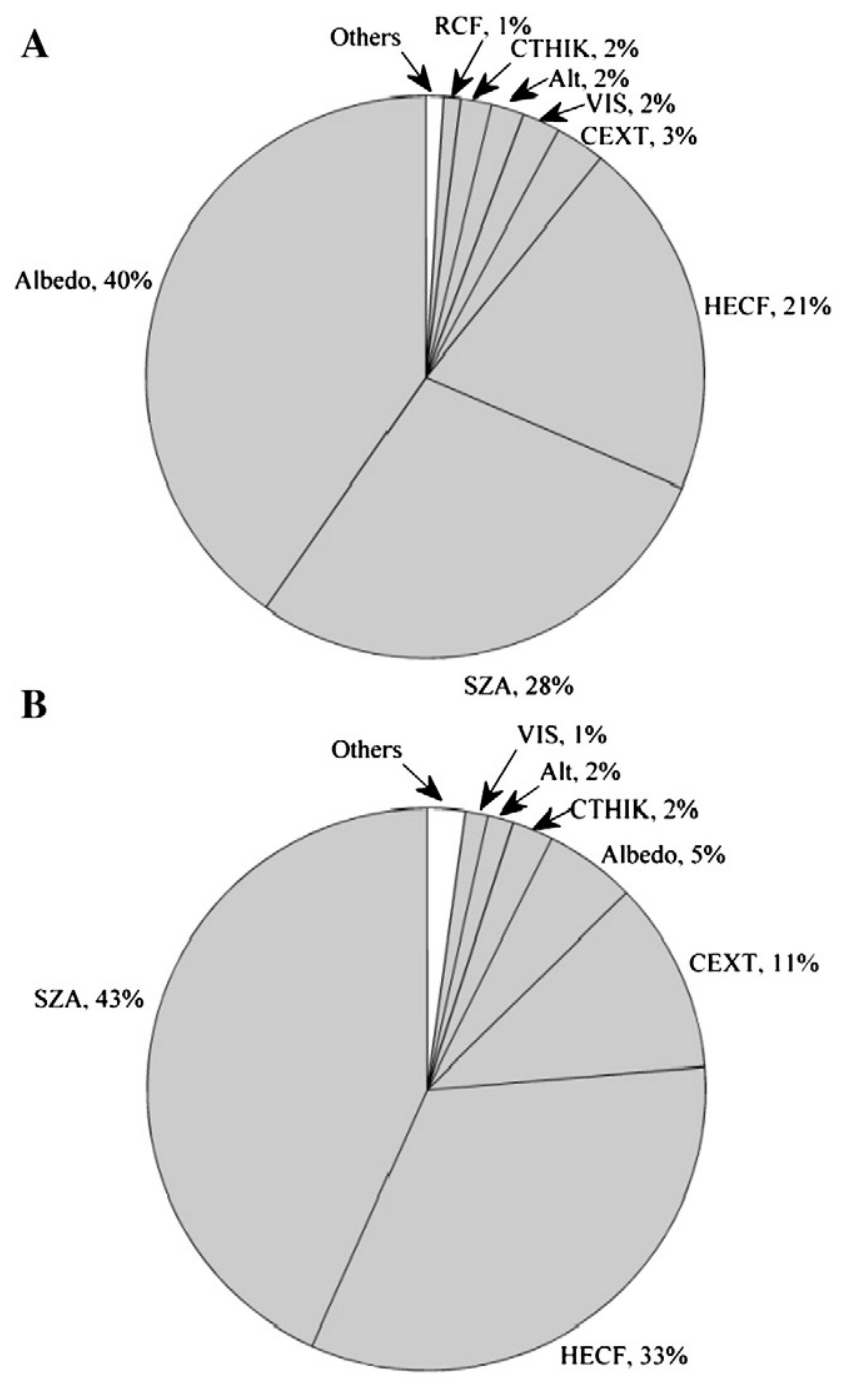

Fig. 8. Normalized total sensitivity index for DSSR with cloudy illumination path obscured by optically thick clouds (A) or optically thin clouds (B).

HECF, CEXT, VIS and Alt) for optically thin clouds (Fig. 11B). The total contribution of Albedo and SZA can reach up to higher than $85 \%$ for both cases. When the illumination direction is obscured by clouds (Fig. 12), the same three parameters (Albedo, SZA and HECF) dominate the USSR. It can be seen that the importance of Albedo for optically thick clouds is higher than that for optically thin clouds, which is the same as DSSR because they both have positive correlation with surface albedo.

The GSA results described above show that 4 out of 12 parameters (33\%) have a null or negligible impact on all the shortwave flux components, including water, ozone, $\mathrm{CO}_{2}$ and CALT. SZA and Alt are identified as important to all components in all situations. SZA ranks top two for all cases, indicating that it is the most influential parameter of the solar radiative transfer model. Comparatively, the importance of Alt is much lower than SZA, and its rankings are higher for clear illumination cases and lower for cloudy illumination cases indicating that the existence of clouds in solar illumination path decreases the effect of Alt on the surface shortwave fluxes. Albedo and HECF are selected as the key parameters to all components other than direct solar flux, since direct solar flux has no relationship with land surface characteristics and it is only influenced by the atmospheric properties in the illumination direction. Albedo ranks first in 9 cases out of 14 (64\%) making it as the second important parameter preceded only by SZA. HECF is the third important parameter in our proposed model since it ranks top three in 11 cases out of 14 (79\%) which is 


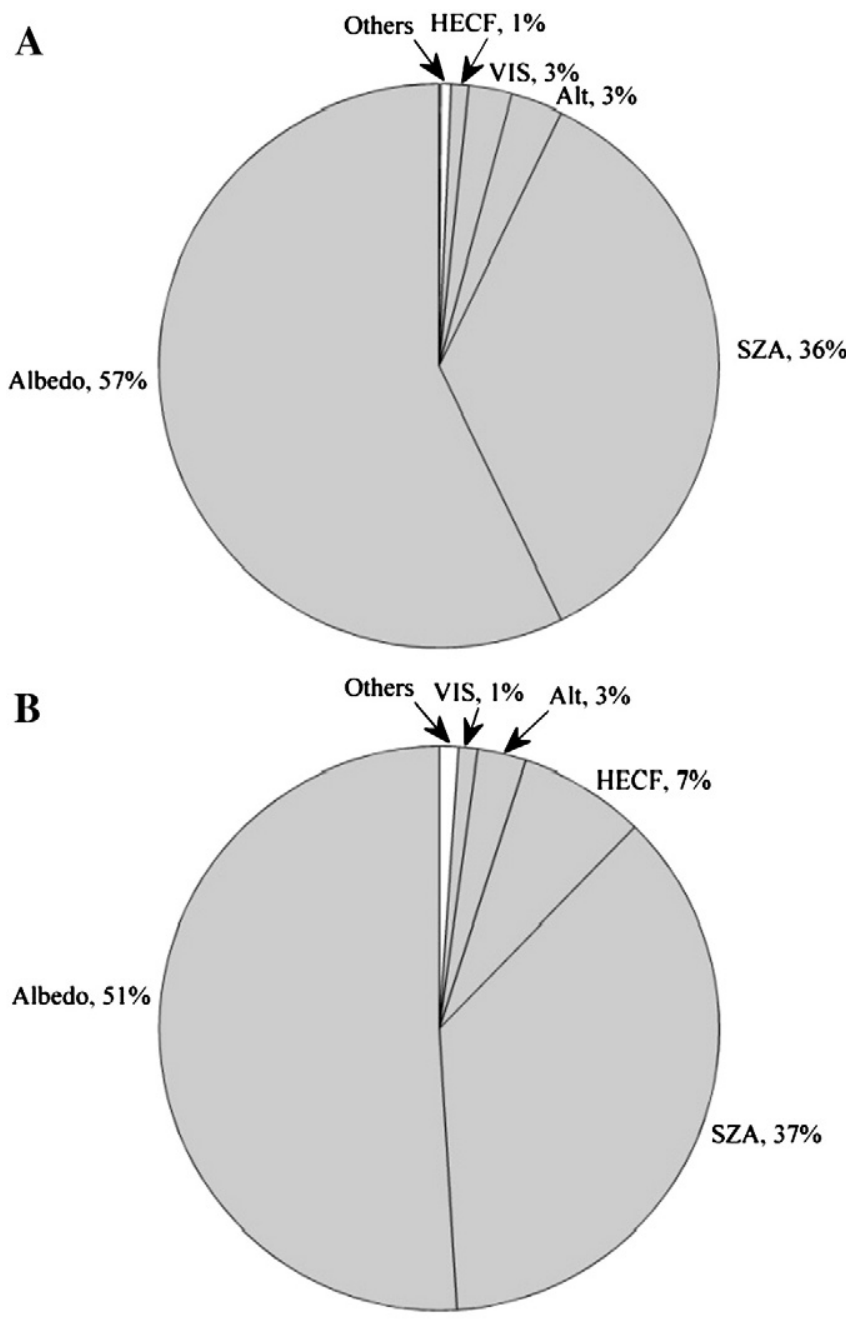

Fig. 9. Normalized total sensitivity index for NSSR with clear illumination path, and optically thick clouds (A) or optically thin clouds (B) might exist in non-illumination hemisphere.

as same as Albedo. The other cloud fraction factor (RCF) involved in the modified model is only slightly important for DSSR (1\%) and NSSR (3\%) when the illumination direction is obscured by optically thick clouds. Two cloud-related parameters including CEXT and CTHIK only play obvious roles when the illumination direction is obscured by clouds, especially by optically thin clouds. When the illumination direction is blocked by optically thick clouds, the two parameters totalized $5 \%$ for the variability of DSSR and $14 \%$ for NSSR. The effect of these parameters can be neglected for all of the clear illumination cases.

Generally speaking, SZA, Albedo, HECF, Alt and VIS are considered as influential to almost all surface shortwave components. Among these five factors, the accuracy of SZA and Alt should be much higher, because SZA is only the function of geographic location and local time, and Alt can be seen as a constant value for a specific place. As an inherent feature of land surface, Albedo can be assumed as constant during a short period (e.g., 8 days). Currently, the combined albedo product of MODIS (MCD43B3) is available globally with the absolute accuracy of 0.02 which should be accurate enough for applications (Salomon et al., 2006). Visibility (VIS) for characterizing aerosol loadings has relatively small effect on almost all shortwave components which indicates that its accuracy might not be strictly demanded. HECF may be the most uncertain factor that might decrease the estimation accuracy of surface shortwave fluxes, because it is a function of cloud base altitude which may be difficult to obtain in operational application.
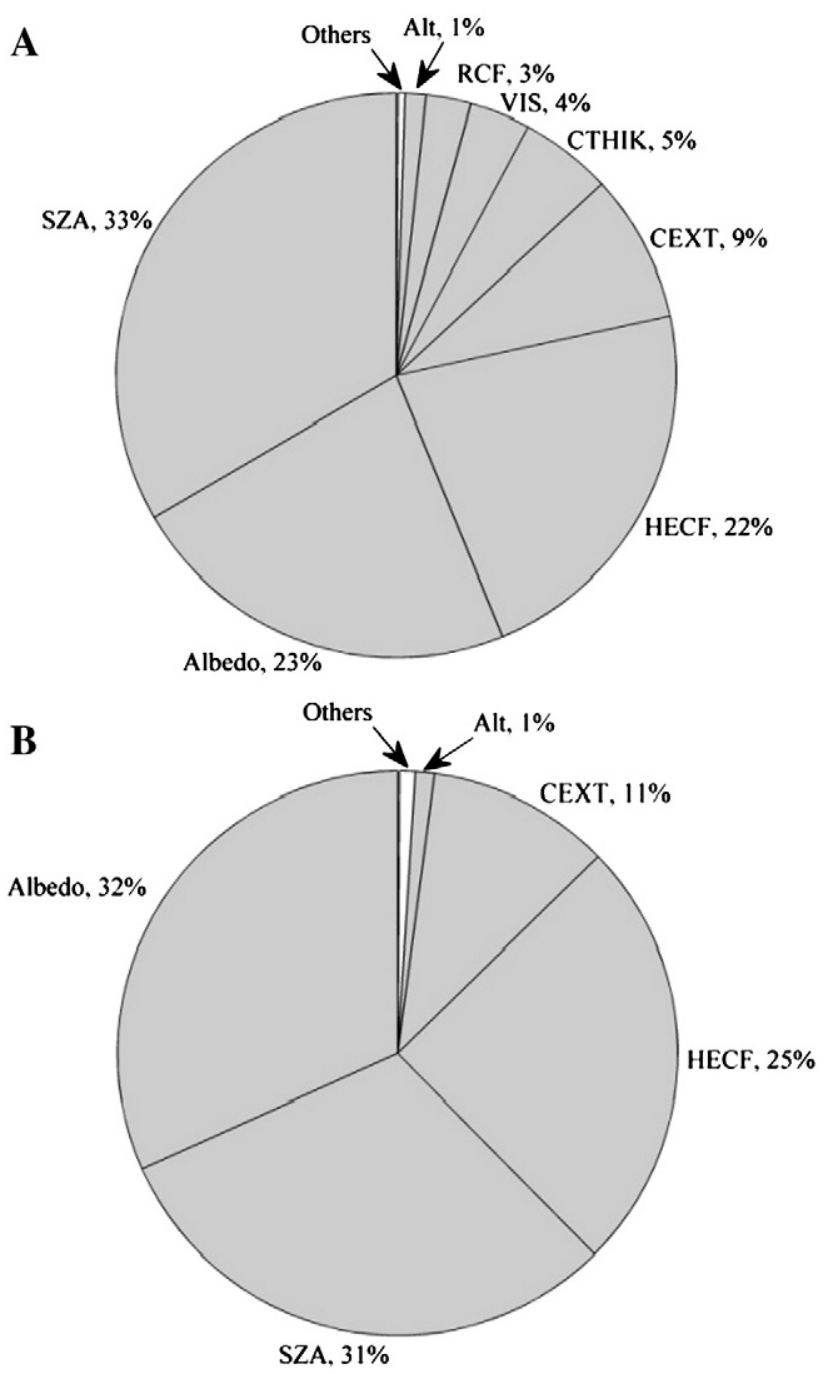

Fig. 10. Normalized total sensitivity index for NSSR with cloudy illumination path obscured by optically thick clouds (A) or optically thin clouds (B).

\section{Comparison and validation}

\subsection{Comparison with the classic MODTRAN by case simulations}

To intuitively illustrate the performance of MODTRAN-CF described above, we compared its predictions for typical atmospheric and surface conditions with that of its prototype (i.e., MODTRAN 4). In the simulations, atmosphere, aerosol and cloud type are fixed as mid-latitude winter, rural and cumulus respectively. Four sets of cloud parameters (CTHIK and CEXT) are chosen in order to represent four levels of cloud extinction which are marked as case 1 to case 4 (see Table 2 ). Case 1 corresponds to optically very thick cloud with highest extinction level, case 2 is also optically thick cloud but with much lower extinction. Case 3 represents optically thin cloud allowing only several units $\left(\mathrm{W} / \mathrm{m}^{2}\right)$ of direct solar flux transmitted directly to the land surface, in contrast, several hundred units of direct solar flux could be transmitted to the surface for optically thin cloud of case 4 . Other parameters are fixed to their nominal values which are also listed in Table 2. The units of all the parameters are given in Table 1. From the GSA results in Section 3, we can see that RCF is much less influential than HECF for the shortwave radiation components of a single point (or a pixel). Since these two factors are not totally independent, especially when they approach extreme values, we assumed they are equal and referred to them as CF in our case simulations. 
A
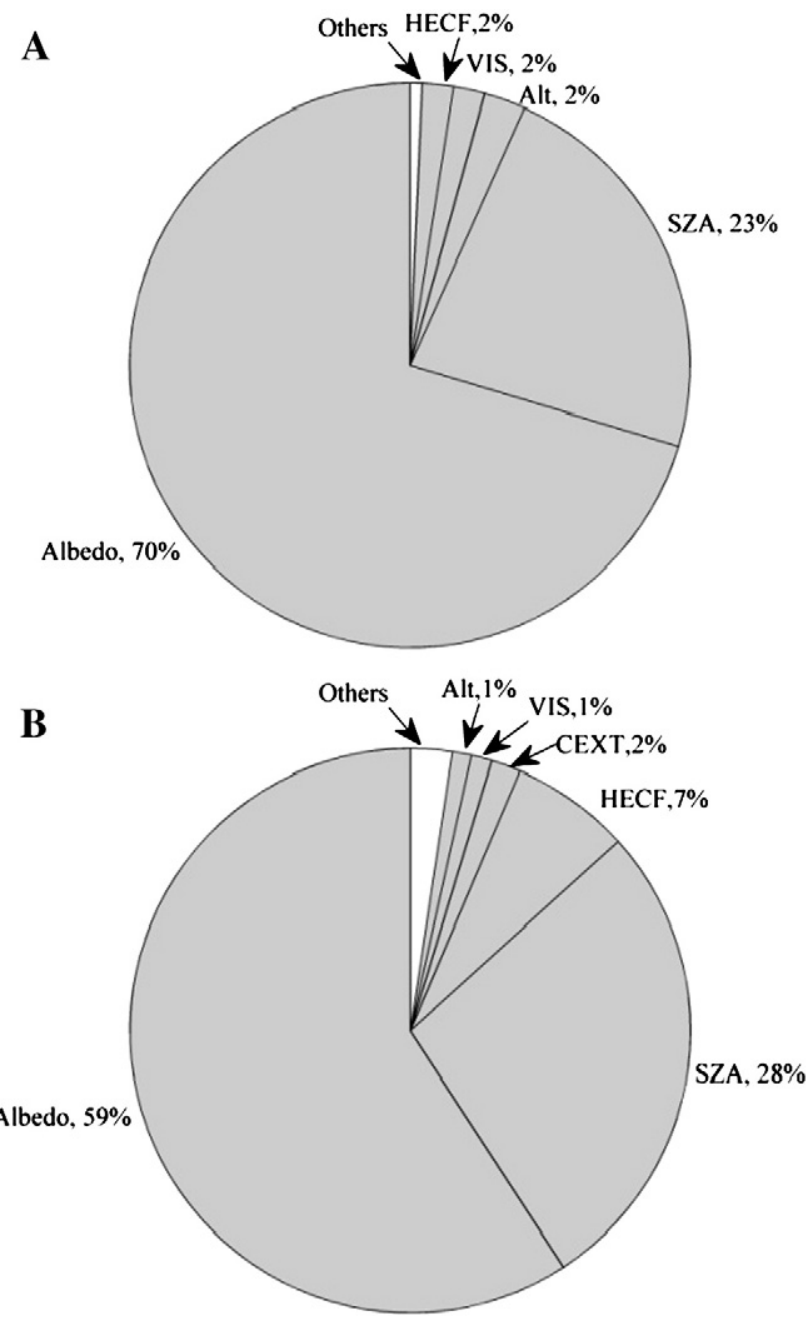

Fig. 11. Normalized total sensitivity index for USSR with clear illumination path, and optically thick clouds (A) or optically thin clouds (B) might exist in non-illumination hemisphere.

Fig. 13 shows that the effect of CF on DSSR should not be ignored in all cases, especially for optically thin clouds. Among the four cases, the downward atmospheric diffuse flux of case 3 is $758 \mathrm{~W} / \mathrm{m}^{2}$ for overcast scenario. The corresponding quantities of case 2 and case 4 are $349 \mathrm{~W} / \mathrm{m}^{2}$ and $594 \mathrm{~W} / \mathrm{m}^{2}$ respectively, and case 1 has the lowest value $\left(106 \mathrm{~W} / \mathrm{m}^{2}\right)$ but is still larger than that of cloud-free scenario which is only $73 \mathrm{~W} / \mathrm{m}^{2}$. From these values, we can easily understand the monotonically increasing relationship between DSSR and CF for almost all cases except case 1 because the relatively small difference between diffuse transmittance of cloud-free and overcast atmosphere of case 1 is more readily canceled out by multiple scattering between land surface and atmosphere. Fig. 13A shows that DSSR is $983 \mathrm{~W} / \mathrm{m}^{2}$ for the cloud-free case, however, if $50 \%$ of the overlying hemisphere other than illumination direction is occupied by clouds, it will increase to $1053 \mathrm{~W} / \mathrm{m}^{2}$ for case $1,1165 \mathrm{~W} / \mathrm{m}^{2}$ for case $2,1338 \mathrm{~W} / \mathrm{m}^{2}$ and $1240 \mathrm{~W} / \mathrm{m}^{2}$ for case 3 and case 4 respectively. The cloud enhancement of diffuse irradiance in clear-sun conditions has not only been investigated by field radiation experiments (e.g., Pfister et al., 2003; Schade et al., 2007), but also has been simulated by 3D radiative transfer models (Segal \& Davis, 1992; Wyser et al., 2002). Pfister et al. (2003) pointed out that the strongest enhancement results from the highest cloud fraction in the case of unobscured sun. Schade et al. (2007) suggested that an excess of more than $500 \mathrm{~W} / \mathrm{m}^{2}$ greater than

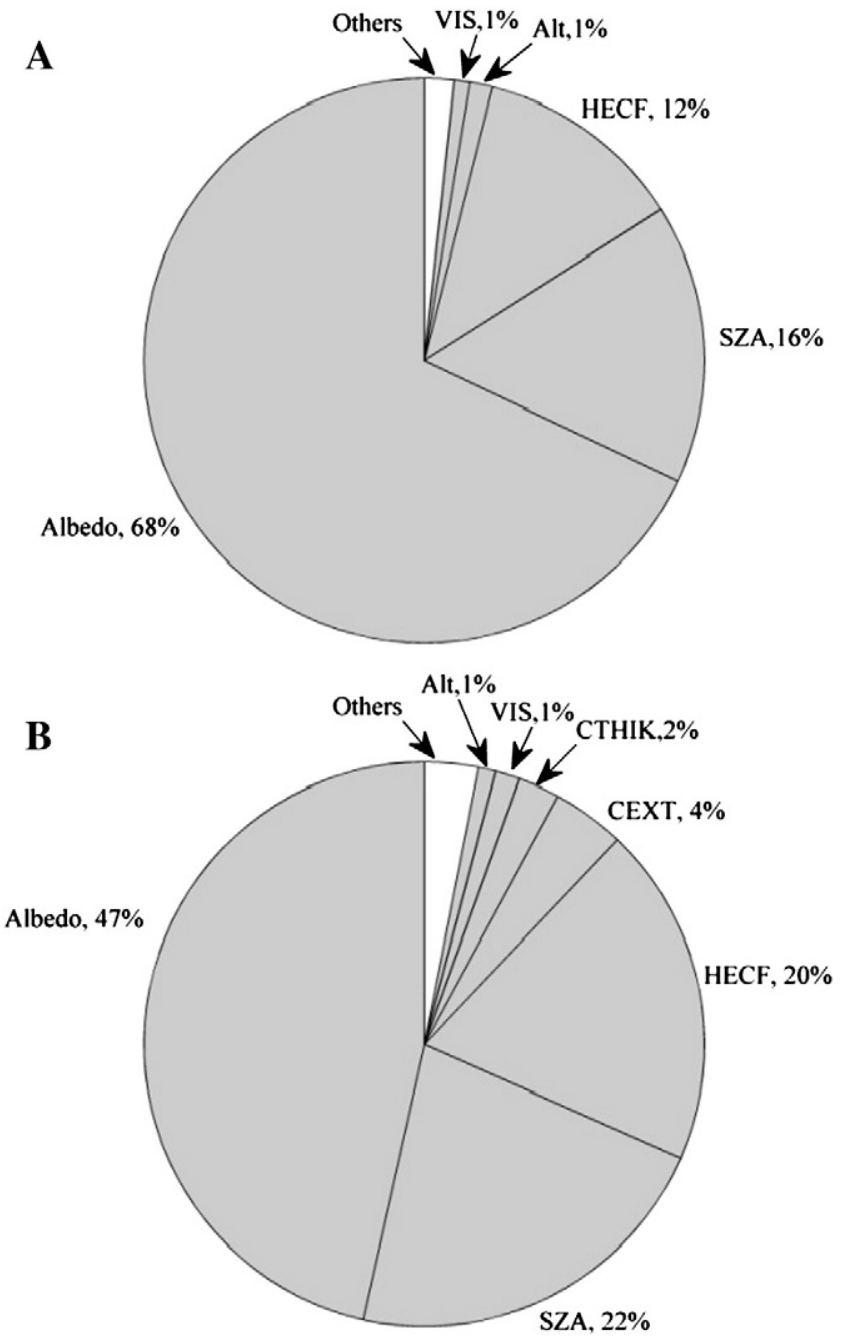

Fig. 12. Normalized total sensitivity index for USSR with cloudy illumination path obscured by optically thick clouds (A) or optically thin clouds (B).

clear sky irradiance is possible. Once the illumination direction is obscured by clouds, the surface direct solar flux is attenuated. The extent of attenuation depends on the extinction level of clouds. Fig. $13 \mathrm{~B}$ shows that DSSR is $135 \mathrm{~W} / \mathrm{m}^{2}$ (case 1 ), $411 \mathrm{~W} / \mathrm{m}^{2}$ (case 2 ), $808 \mathrm{~W} / \mathrm{m}^{2}$ (case 3 ) and $957 \mathrm{~W} / \mathrm{m}^{2}$ (case 4 ) respectively for the overcast scenarios. When the overlying hemisphere other than illumination direction is $50 \%$ clear, those values change to $166,278,459$ and $700 \mathrm{~W} / \mathrm{m}^{2}$ respectively.

Fig. 14 shows the TOA radiance of MODIS band $1(620-670 \mathrm{~nm})$ as a function of $\mathrm{CF}$ in all situations illustrated in Fig. 2 for the four cloud

Table 2

Values of parameters fixed in the cases of simulation.

\begin{tabular}{llllllll}
\hline VZA $^{\text {a }}$ & RAA $^{\mathrm{a}}$ & SZA & Albedo & VIS & Alt & CALT & $\mathrm{CO}_{2}$ \\
\hline 30 & 180 & 30 & 0.3 & 23 & 4 & 0.7 & 375 \\
CTHIK & & & & CEXT & & & \\
Case 1 & Case 2 & Case 3 & Case 4 & Case 1 & Case 2 & Case 3 & Case 4 \\
1 & 1 & 0.5 & 0.1 & 80 & 20 & 8 & 8 \\
\hline
\end{tabular}

a VZA and RAA represent viewing zenith angle and relative azimuth angle respectively. Their units are the same as SZA in Table 1. 

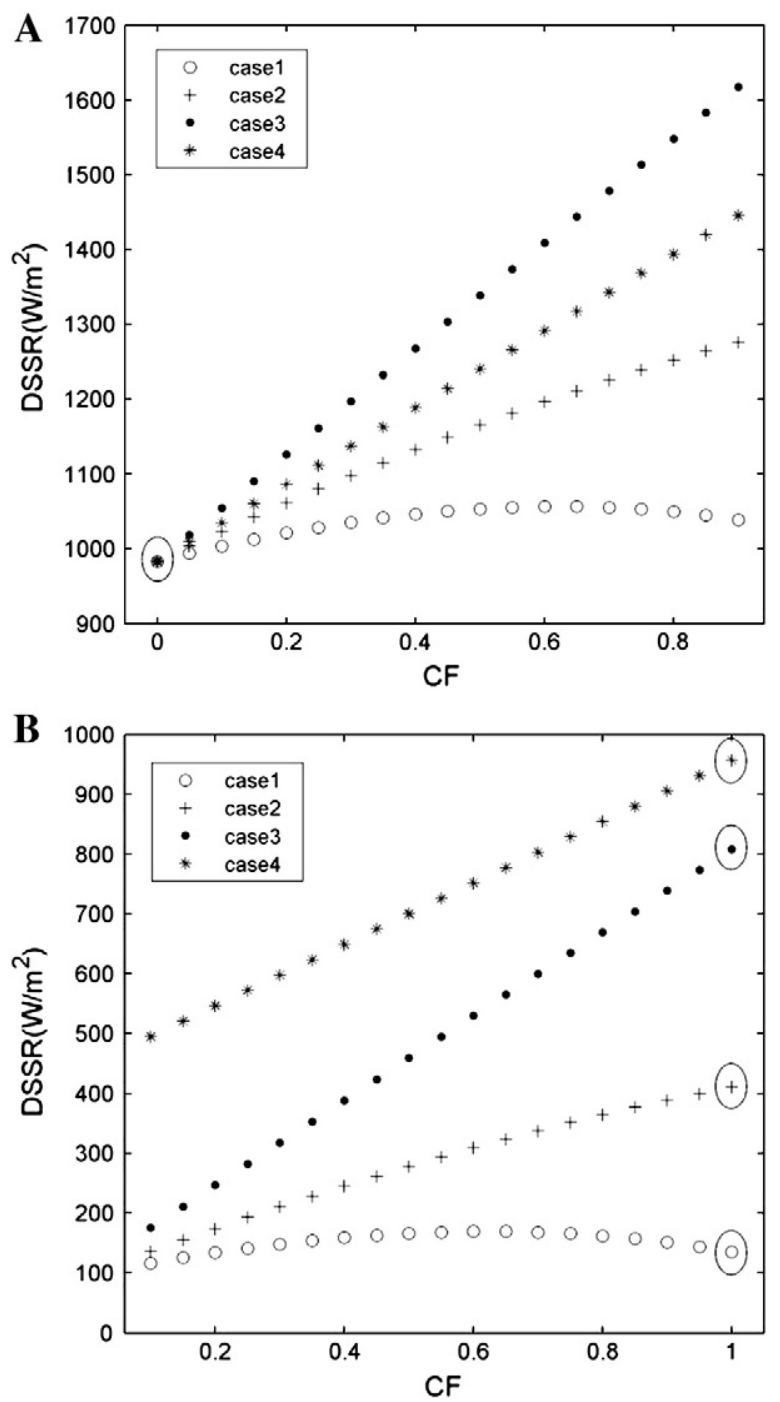

Fig. 13. DSSR of a pixel as a function of $C F$ for clear illumination (A) and cloudy illumination (B) situations, values of MODTRAN are highlighted by ellipses.

cases described above. Similar patterns were found for other bands of MODIS or other sensors (not shown). Among the four cases, the cloud of case 1 is optically very thick cloud with highest reflectivity, lowest diffuse and no direct transmittance, so in Fig. 14A and D, the TOA radiance of case 1 is essentially constituted by path radiance. Therefore, the TOA radiance of case 1 in Fig. 14A is slightly larger than that in Fig. 14D with only $10 \mathrm{~W} / \mathrm{m}^{2}$ due to the slightly higher diffuse transmission for the situation of Fig. 14A with clear illumination. Compared to case 1 , the TOA radiances of case 2 in Fig. $14 \mathrm{~A}$ and $\mathrm{D}$ have lower values owing to its smaller reflectivity. In addition, its higher diffuse transmittance enhances the surface contribution, which makes the TOA radiance difference of case 2 between Fig. 14A and D slightly larger than that of case 1 . Lower reflectivity and higher transmittance of optically thin clouds lead to smaller path radiance and higher surface contribution for cases 3 and 4 . Compared to case 4, the surface contribution of case 3 is higher under clear illumination conditions (Fig. 13A) but lower under cloudy illumination cases (Fig. 13B), in combination with its higher path radiance, we can easily understand the larger difference in Fig. 14A but smaller difference in Fig. 14D between case 3 and case 4. The TOA radiance is mainly contributed by radiation from the surface for the cases of clear viewing path, which explains the significant difference between TOA radiance in Fig. 14B and C, and also explains the remarkable resemblance between Figs. 14B and 13B, and those between Figs. 14C and 13A.

Based on the analysis performed above, we can conclude that it necessary to introduce HECF into the simulation of surface shortwave radiation and the TOA radiance with $1 \mathrm{D}$ plane-parallel radiative transfer model and that the simulation results of MODTRAN-CF are theoretically reasonable.

\subsection{Validation with field measurements}

A straightforward validation of our physical model with field measurement data is almost impossible, since many inputs are not readily available. For example, the information of atmospheric profile, surface reflectance, aerosol type, visibility, cloud type, cloud base height, cloud thickness, cloud extinction coefficient and hemispherical cloud cover, are all needed to run the MODTRAN-CF of the present study. Only a limited number of field sites around the world can provide such comprehensive measurements. As described above, the essential difference between MODTRAN-CF and MODTRAN is the involvement of cloud fraction, especially the hemispherical cloud cover, so field radiation measurements and collocated hemispherical cloud cover are the basic materials for validation. Other inputs can be determined under reasonable hypothesis.

In Lauder, Central Otago, New Zealand $\left(45.038^{\circ} \mathrm{S}, 169.684^{\circ} \mathrm{E}\right.$; altitude, $370 \mathrm{~m}$ ), a total sky imager (TSI) began operating in September 1999, measuring the hemispherical cloud cover once per minute as well as indicating whether the sun is obscured by clouds. The 1-min average values of global, direct and diffuse surface solar irradiance over the spectral interval from 0.3 to $3.0 \mu \mathrm{m}$ have also been observed by radiation sensors as part of the international Baseline Surface Radiation Network (BSRN) since August 1999. The two different sets of equipment are both located on the roof of a laboratory building with a separation of only $5 \mathrm{~m}$. For detailed information about the equipment, please refer to Pfister et al. (2003) and Ohmura et al. (1998).

The ability of capturing cloud-related solar radiation enhancement is the most obvious superiority of MODTRAN-CF. Fig. 15A shows an example of the measured DSSR and its direct and diffuse components for the solar radiation enhanced time period of 16 December, 2000 (around 1500 NZST). Regarding the inputs for the model, midlatitude summer atmosphere is selected given the site geolocation and the measurement date. According to the very intense direct irradiance under clear sky (Fig. 15A), no aerosol effect is included in the following simulation, which is in agreement with the typical low aerosol loading at this site. The surface albedo is set to a nominal value of 0.3 since the site is surrounded by grassland (McKenzie et al., 1996). Manual inspection of the TSI images suggests cumulus clouds around the position of the sun during the enhanced time period (Pfister et al., 2003). Since the specific cloud characteristics are not known, we set three values to indicate different levels of cloud effects. Specifically, cloud thickness and cloud base height are set to $1.5 \mathrm{~km}$, while the cloud extinction coefficient is set to $40 \mathrm{~km}^{-1}, 10 \mathrm{~km}^{-1}$ and $5 \mathrm{~km}^{-1}$ respectively. For these cloud settings, no direct solar flux will be detected on the ground when the sun disk is totally cloud covered. It can be seen from Fig. 15A that the reduction of direct solar flux may be caused by clouds that partially cover the sun disk, so the fraction of sun disk occulted by clouds must be computed for each moment before the simulation. The main steps to calculate the fraction of sun disk occulted by clouds are as follows: (1) fit an empirical linear relationship between clear-sky direct solar flux and the cosine of solar zenith angle; (2) calculate the expected clear-sky direct solar flux during the period using the empirical relationship; (3) compute the fraction of sun disk occulted by clouds as a function of the real direct solar flux and the expected clear-sky values (Fig. 15B).

Because some hypothetical conditions may not be consistent with the real cases, it is difficult to match the real values of the simulation 

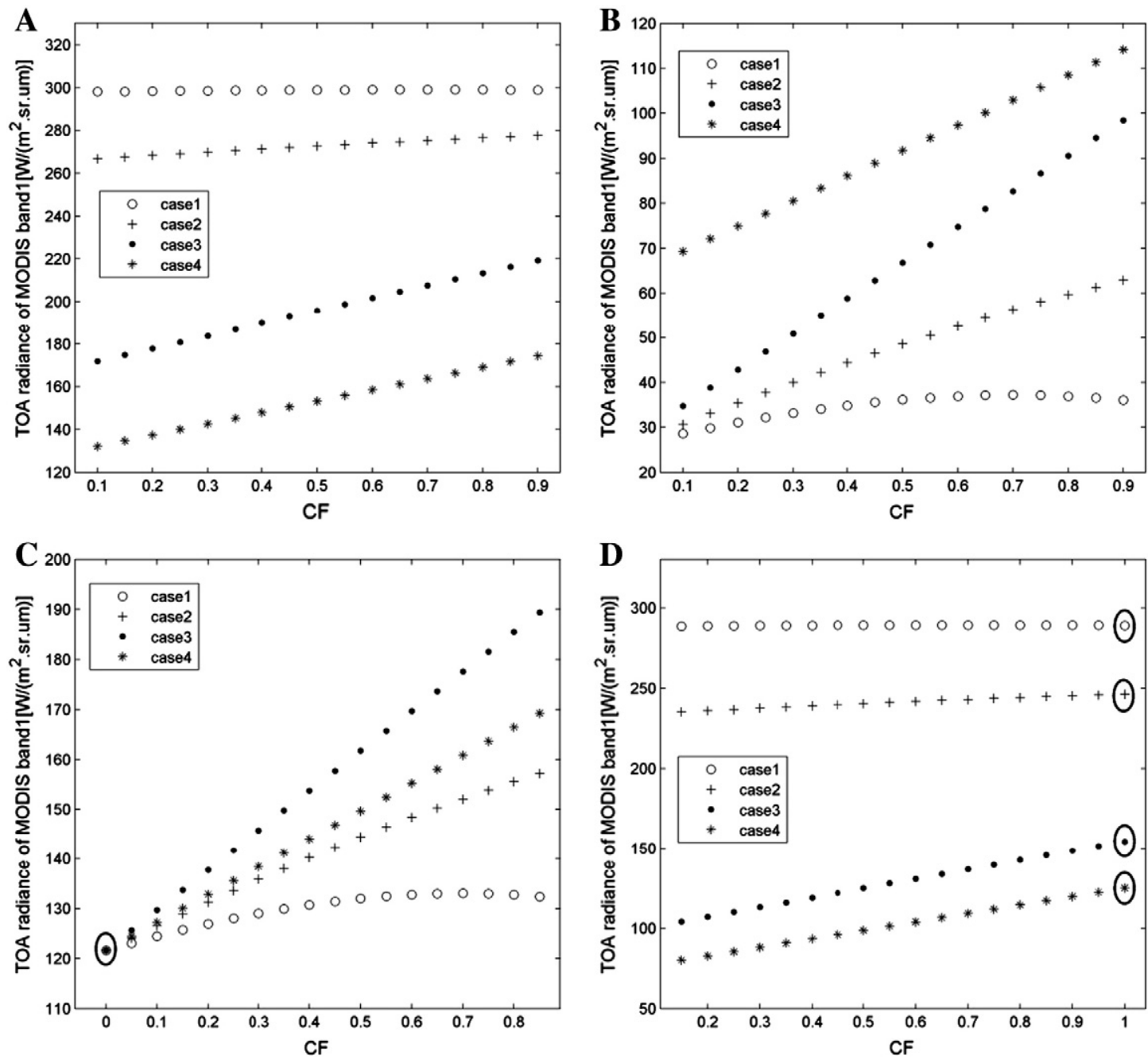

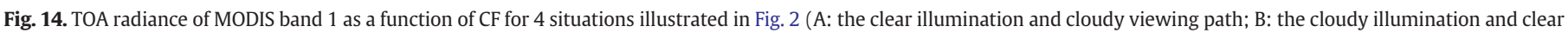

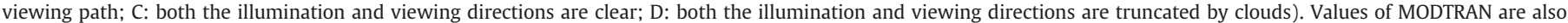
highlighted by ellipses.

and the field measurements. However, the superiority of MODTRANCF is obviously demonstrated by the simulation results (Fig. 16), in which the variation patterns fit the real cases (Fig. 15A) very well. The extent of approximation is mainly determined by the cloud scattering intensity.

\section{Conclusion}

Clouds, with their particular spatial and temporal variability, are considered to be the strongest modulator of the solar radiant energy absorbed by the earth-atmosphere system. Traditional 1D planeparallel radiative transfer models can only consider two ideal situations: cloud-free and overcast cases. In this paper, an improved model named as MODTRAN-CF has been proposed based on the classic 1D radiative transfer model (i.e., MODTRAN 4) with the incorporation of hemispherical effective cloud fraction (HECF) and regional cloud fraction (RCF). MODTRAN-CF has the ability to simulate surface shortwave radiation budget and TOA radiance for all possible solar-cloud-viewing geometries with reasonable simulation results in theory. Its accuracy is dependent on its prototype (MODTRAN) which has been widely used and validated in radiative transfer modeling. An advantage of this approach is that it does not require significant portions of the existing radiative transfer code to be rewritten. The superiority of MODTRAN-CF has been validated using some field measurements from an unpolluted BSRN site in New Zealand. The validation results indicate that the variation patterns of DSSR under partially cloudy conditions fit the real cases very well. Although more validation activities are in progress, we feel confident at this point that MODTRAN-CF is more superior for retrieving surface shortwave radiation components under partially cloudy conditions.

Sensitivity analysis of model parameters is a fundamental prerequisite for correctly understanding and further simplifying physical models. In light of this, one of the most representative GSA methods was used to analyze the effect of model parameters on the surface shortwave radiation components. However, we must note that the GSA results may depend on the distributions and ranges of model parameters, and the sensitivity of model outputs can be influenced by the predefined conditions of simulation such as the types of atmosphere, aerosol and cloud. Therefore, the sensitivity analysis results described above might not be considered universal and the simulation conditions should be changed substantially according to specific model application.

This more physically plausible solar radiative transfer model and its GSA results will support us to create more accurate statistical or parameterized relationships between key factors and target radiation 

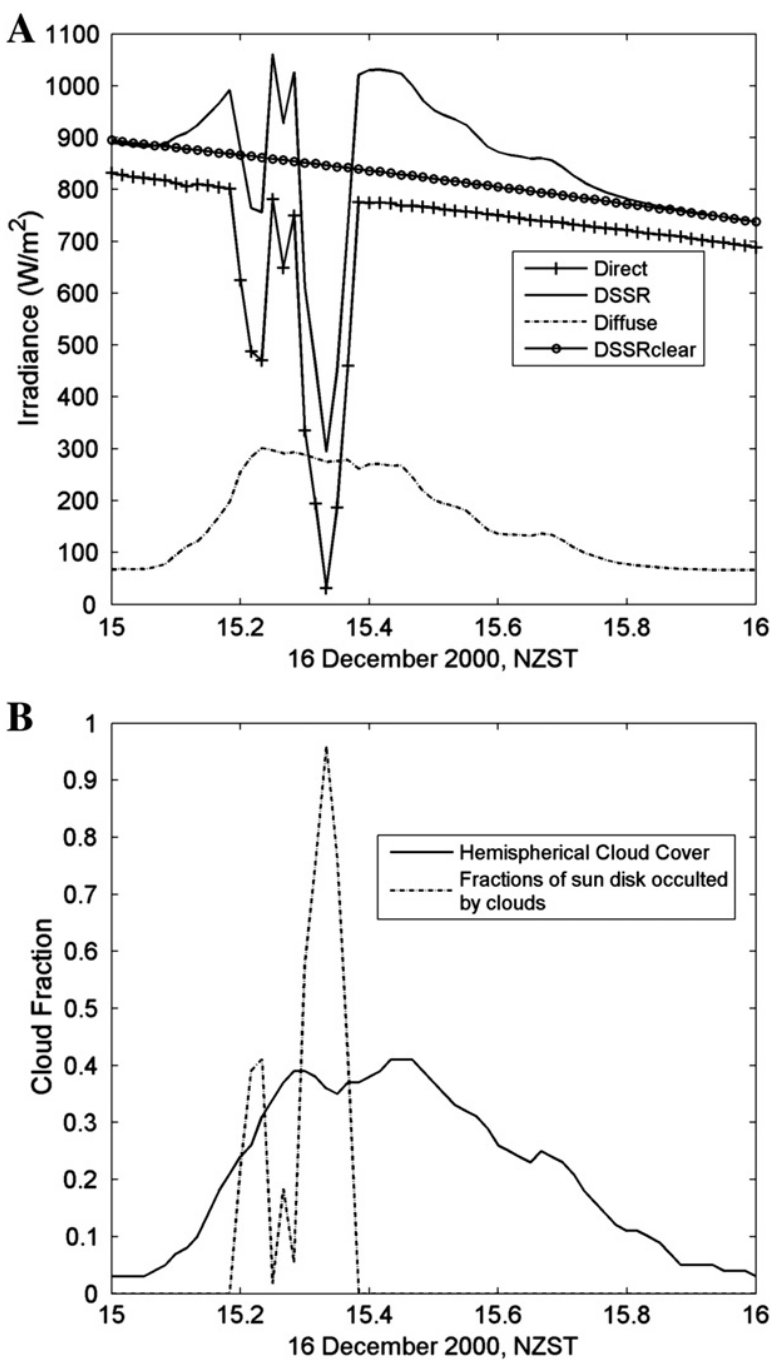

Fig. 15. (A) Field measured DSSR and its direct and diffuse components for the solar radiation enhanced time period of 16 December, 2000. DSSRclear is the empirical fitted clear sky DSSR as function of the solar zenith angle. (B) The hemispherical cloud cover retrieved by TSI during the enhanced time period and the calculated fractions of sun disk occulted by clouds.

components. The application and validation of MODTRAN-CF and its corresponding parameterized algorithms for each shortwave radiation component will be shown in a companion paper.

\section{Acknowledgments}

This work has been supported by the National Natural Science Foundation of China (Grant No. 40871164) and the European Commission (Call FP7-ENV-2007-1 Grant No. 212921) as part of the CEOP-AEGIS project (http://www.ceop-aegis.org/) coordinated by the Universite de Strasbourg. The field measurements were provided by NIWA, NDACC, and BSRN. The authors would like to thank Prof. Shunlin Liang and Dr. Dongdong Wang for their help on the understanding of cloud models in MODTRAN, Ying Sun from the University of Texas and Tao He from the University of Maryland for their comments on the original manuscript, and three anonymous reviewers for their valuable comments that have greatly improved the presentation of this paper.
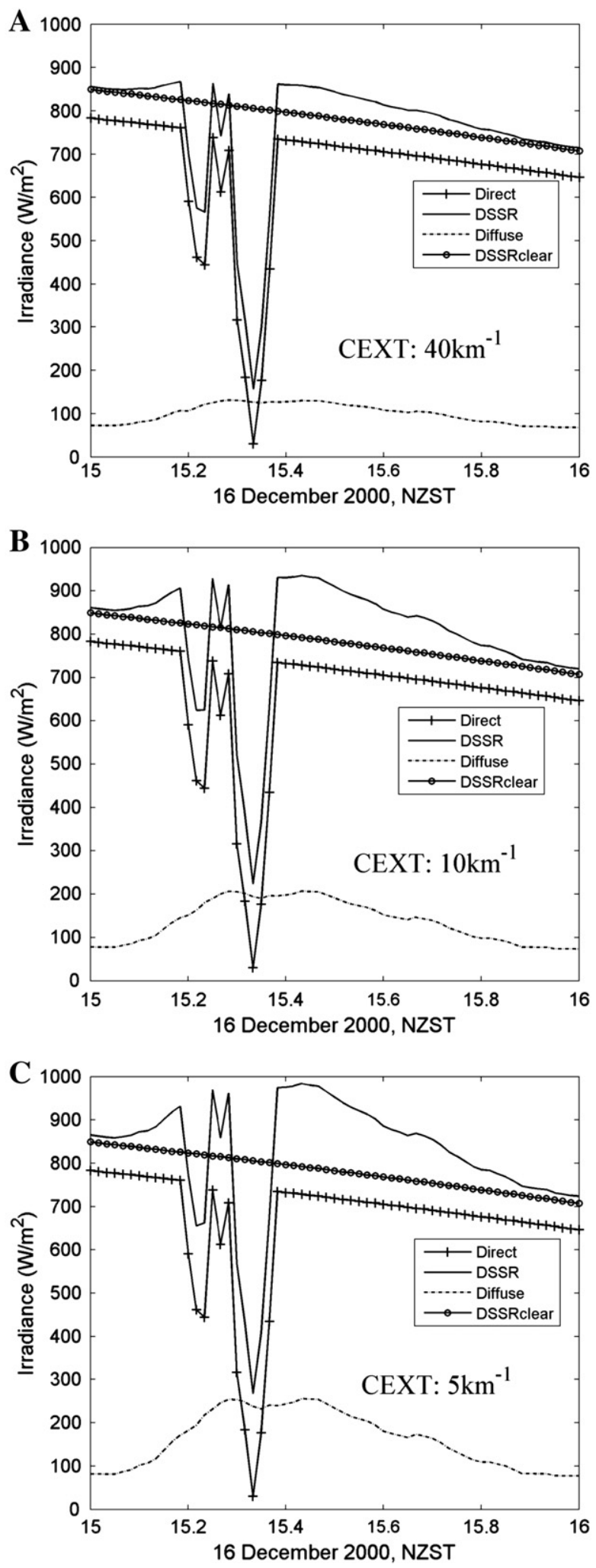

Fig. 16. Simulation results of MODTRAN-CF for the solar radiation enhanced time period with different sets of cloud extinction coefficient. DSSRclear is the output of MODTRAN 4 under clear sky.

\section{References}

Arking, A. (1991). The radiative effects of clouds and their impact on climate. Bulletin of the American Meteorological Society, 72(6), 795-953.

Barker, H. W. (1996). Estimating cloud field albedo using one-dimensional series of optical depth. Journal of the Atmospheric Sciences, 53(19), 2826-2837. 
Barker, H. W., Stephens, G. L., \& Fu, Q. (1999). The sensitivity of domain-averaged solar fluxes to assumptions about cloud geometry. Quarterly Journal of the Royal Meteorological Society, 125(558), 2127-2152.

Butt, N., New, M., Malhi, Y., Da Costa, A. C. L., Oliveira, P., \& Silva-Espejo, J. E. (2010). Diffuse radiation and cloud fraction relationships in two contrasting Amazonian rainforest sites. Agricultural and Forest Meteorology, 150(3), 361-368.

Cahalan, R. F., Oreopoulos, L., Marshak, A., Evans, K., Davis, A. B., Pincus, R., et al. (2005) The International Intercomparison of 3D Radiation Codes (I3RC): Bringing together the most advanced radiative transfer tools for cloudy atmospheres. Bulletin of the American Meteorological Society, 86(9), 1275-1293.

Cahalan, R. F., Ridgway, W., Wiscombe, W. J., \& Gollmer, S. (1994). Independent pixel and Monte Carlo estimates of stratocumulus albedo. Journal of the Atmospheric Sciences, 51(24), 3776-3790.

Cariboni, J., Gatelli, D., Liska, R., \& Saltelli, A. (2007). The role of sensitivity analysis in ecological modelling. Ecological Modelling, 203(1-2), 167-182.

Ceballos, J. C., Bottino, M. J., \& de Souza, J. M. (2004). A simplified physical model for assessing solar radiation over Brazil using GOES 8 visible imagery. Journal of Geophysical Research, 109, D02211.

Confalonieri, R. (2011). Combining a weather generator and a standard sensitivity analysis method to quantify the relevance of weather variables on agrometeorological models outputs. Theoretical and Applied Climatology, 108(1), 19-30.

Confalonieri, R., Bellocchi, G., Bregaglio, S., Donatelli, M., \& Acutis, M. (2010). Comparison of sensitivity analysis techniques: A case study with the rice model WARM. Ecological Modelling, 221(16), 1897-1906.

Cornet, C., C-Labonnote, L., \& Szczap, F. (2010). Three-dimensional polarized Monte Carlo atmospheric radiative transfer model (3DMCPOL): 3D effects on polarized visible reflectances of a cirrus cloud. Journal of Quantitative Spectroscopy and Radiative Transfer, 111(1), 174-186.

Deneke, H., Feijt, A., van Lammeren, A., \& Simmer, C. (2005). Validation of a physical retrieval scheme of solar surface irradiances from narrowband satellite radiances. Journal of Applied Meteorology, 44(9), 1453-1466.

Diak, G. R., \& Gautier, C. (1983). Improvements to a simple physical model for estimating insolation from GOES data. Journal of Applied Meteorology, 22(3), 505-508.

Drouet, J. L., Capian, N., Fiorelli, J. L., Blanfort, V., Capitaine, M., Duretz, S., et al. (2011). Sensitivity analysis for models of greenhouse gas emissions at farm level. Case study of $\mathrm{N}_{2} \mathrm{O}$ emissions simulated by the CERES-EGC model. Environmental Pollution, 159(11), 3156-3161.

Dubayah, R. (1992). Estimating net solar radiation using landsat thematic mapper and digital elevation data. Water Resources Research, 28(9), 2469-2484.

Evans, K. F. (1998). The spherical harmonics discrete ordinate method for three-dimensional atmospheric radiative transfer. Journal of the Atmospheric Sciences, 55(3), 429-446.

Freidenreich, S. M., \& Ramaswamy, V. (1999). A new multiple-band solar radiative parameterization for general circulation models. Journal of Geophysical Research, 104(D24), 31389-31409.

Frouin, R., \& Chertock, B. (1992). A technique for global monitoring of net solar irradiance at the ocean surface. Part I: Model. Journal of Applied Meteorology, 31(9), $1056-1066$.

Gautier, C., Diak, G., \& Masse, S. (1980). A simple physical model to estimate incident solar radiation at the surface from GOES satellite data. Journal of Applied Meteorology, 19(8), 1005-1012.

Gautier, C., \& Landsfeld, M. (1997). Surface solar radiation flux and cloud radiative forcing for the Atmospheric Radiation Measurement (ARM) Southern Great Plains (SGP): A satellite, surface observations, and radiative transfer model study. Journal of the Atmospheric Sciences, 54(10), 1289-1307.

Gu, Y., \& Liou, K. N. (2001). Radiation parameterization for three-dimensional inhomogeneous cirrus clouds: Application to climate models. Journal of Climate, 14(11), 2443-2457.

Hartmann, D. L., Ramanathan, V., Berroir, A., \& Hunt, G. E. (1986). Earth radiation budget data and climate research. Reviews of Geophysics, 24(2), 439-468.

Kim, H.Y. (2008). Estimation of land surface radiation budget from MODIS data. Ph.D dissertation, Department of Geography, University of Maryland.

Lam, J. C., \& Li, D. H. W. (1996). Correlation between global solar radiation and its direct and diffuse components. Building and Environment, 31(6), 527-535.

Li, Z., Barker, H. W., \& Moreau, L. (1995). The variable effect of clouds on atmospheric absorption of solar radiation. Nature, 376(10), 486-490.

Li, Z., Leighton, H. G., Masuda, K., \& Takashima, T. (1993). Estimation of SW flux absorbed at the surface from TOA reflected flux. Journal of Climate, 6(2), 317-330.

Liang, S. (2004). Quantitative remote sensing of land surfaces. New York: John Wiley \& Sons $534 \mathrm{pp}$.

Liang S, Wang, K., Wang, W., Wang, D., Gui, S, Zhang, X et al. (2009). Mapping high-resolution land surface radiative fluxes from MODIS: Algorithms and preliminary validation results. Geospatial Technology for Earth Observation, 141-176.

Liang, S., Zheng, T., Liu, R., Fang, H., Tsay, S. -C., \& Running, S. (2006). Estimation of incident photosynthetically active radiation from moderate resolution imaging spectrometer data. Journal of Geophysical Research, 111, D15208.
Liu, R., Liang, S., He, H., Liu, J., \& Zheng, T. (2008). Mapping incident photosynthetically active radiation from MODIS data over China. Remote Sensing of Environment, 112(3), 998-1009.

Marshak, A., \& Davis, A. B. (2005). 3D radiative transfer in cloudy atmospheres. Berlin: Springer $686 \mathrm{pp}$.

Masuda, K., Leighton, H. G., \& Li, Z. (1995). A new parameterization for the determination of solar flux absorbed at the surface from satellite measurements. Journal of Climate, 8(6), 1615-1629.

McKenzie, R. L., Kotkamp, M., \& Ireland, W. (1996). Upwelling UV spectral irradiances and surface albedo measurements at Lauder, New Zealand. Geophysical Research Letters, 23(14), 1757-1760.

Monteith, J. L. (1975). Vegetation and the atmosphere. Volume 1: Principles. : Academic Press $278 \mathrm{pp}$

O'Hirok, W., \& Gautier, C. (1998). A three-dimensional radiative transfer model to investigate the solar radiation within a cloudy atmosphere. Part I: Spatial effects. Journal of the Atmospheric Sciences, 55(12), 2162-2179.

Ohmura, A., Gilgen, H., Hegner, H., Müller, G., Wild, M., Dutton, E. G., et al. (1998). Baseline surface radiation network (BSRN/WCRP): New precision radiometry for climate research. Bulletin of the American Meteorological Society, 79(10), 2115-2136.

Pfister, G., McKenzie, R. L., Liley, J. B., Thomas, A., Forgan, B. W., \& Long, C. N. (2003). Cloud coverage based on all-sky imaging and its impact on surface solar irradiance. Journal of Applied Meteorology, 42(10), 1421-1434.

Pinker, R. T., \& Ewing, J. A. (1985). Modeling surface solar radiation: Model formulation and validation. Journal of Applied Meteorology, 24(5), 389-401.

Pinker, R. T., \& Laszlo, I. (1992). Modeling surface solar irradiance for satellite applications on a global scale. Journal of Applied Meteorology, 31(2), 194-211.

Richter, G. M., Acutis, M., Trevisiol, P., Latiri, K., \& Confalonieri, R. (2010). Sensitivity analysis for a complex crop model applied to Durum wheat in the Mediterranean. European Journal of Agronomy, 32(2), 127-136.

Salomon, J. G., Schaaf, C. B., Strahler, A. H., Gao, F., \& Jin, Y. (2006). Validation of the MODIS bidirectional reflectance distribution function and albedo retrievals using combined observations from the aqua and terra platforms. IEEE Transactions on Geoscience and Remote Sensing, 44(6), 1555-1565.

Saltelli, A., Tarantola, S., \& Campolongo, F. (2000). Sensitivity analysis as an ingredient of modeling. Statistical Science, 15(4), 377-395.

Schade, N. H., Macke, A., Sandmann, H., \& Stick, C. (2007). Enhanced solar global irradiance during cloudy sky conditions. Meteorologische Zeitschrift, 16(3), 295-303.

Segal, M., \& Davis, J. (1992). The impact of deep cumulus reflection on the ground-level global irradiance. Journal of Applied Meteorology, 31(2), 217-222.

Simlab (2011). Software package for uncertainty and sensitivity analysis. Joint Research Centre of the European Commission. Downloadable for free at: http:// simlab.jrc.ec.europa.eu

Sobol', I. M. (1993). Sensitivity analysis for non-linear mathematical models. Mathematical Modelling and Computational Experiment, 1, 407-414.

Sobol', I. M. (2001). Global sensitivity indices for nonlinear mathematical models and their Monte Carlo estimates. Mathematics and Computers in Simulation, 55(1-3), $271-280$.

Stamnes, K., \& Tsay, S. (1988). Numerically stable algorithm for discrete-ordinate-method radiative transfer in multiple scattering and emitting layered media. Applied Optics, 27(12), 2502-2509.

Tang, B., Li, Z. -L., \& Zhang, R. (2006). A direct method for estimating net surface shortwave radiation from MODIS data. Remote Sensing of Environment, 103(1), 115-126.

Woods, J. D., Barkmann, W., \& Horch, A. (1984). Solar heating of the oceans-Diurnal, seasonal and meridional variation. Quarterly Journal of the Royal Meteorological Society, 110(465), 633-656.

Wyser, K., O'Hirok, W., \& Gautier, C. (2005). A simple method for removing 3-D radiative effects in satellite retrievals of surface irradiance. Remote Sensing of Environment, 94(3), 335-342.

Wyser, K., O'Hirok, W., Gautier, C., \& Jones, C. (2002). Remote sensing of surface solar irradiance with corrections for 3-D cloud effects. Remote Sensing of Environment, 80(2), 272-284.

Zhang, Y., Rossow, W. B., Lacis, A. A., Oinas, V., \& Mishchenko, M. I. (2004). Calculation of radiative fluxes from the surface to top of atmosphere based on ISCCP and other global data sets: Refinements of the radiative transfer model and the input data. Journal of Geophysical Research, 109, D19105.

Zheng, T. (2007). Mapping photosynthetically active radiation (PAR) using multiple remote sensing data. Ph.D dissertation, Department of Geography, University of Maryland.

Zuidema, P., \& Evans, K. F. (1998). On the validity of the independent pixel approximation for boundary layer clouds observed during ASTEX. Journal of Geophysical Research, 103(D6), 6059-6074. 